TRANSACTIONS OF THE

AMERICAN MATHEMATICAL SOCIETY

Volume 362, Number 8, August 2010, Pages 4161-4190

S 0002-9947(10)04945-7

Article electronically published on March 24, 2010

\title{
HOMOGENIZATION OF ONE-PHASE STEFAN-TYPE PROBLEMS IN PERIODIC AND RANDOM MEDIA
}

\author{
INWON C. KIM AND ANTOINE MELLET
}

\begin{abstract}
We investigate the homogenization of Stefan-type problems with oscillating diffusion coefficients. Both cases of periodic and random (stationary ergodic) mediums are considered. The proof relies on the coincidence of viscosity solutions and weak solutions (which are the time derivatives of the solutions of an obstacle problem) for the Stefan problem. This coincidence result is of independent interest.
\end{abstract}

\section{INTRODUCTION}

Let $v_{0}(x)$ be a nonnegative function defined in $\mathbb{R}^{n}$ with compact support $\Omega_{0}=$ $\left\{v_{0}>0\right\}$. This paper is concerned with one-phase Stefan problems of the form:

$$
\begin{cases}v_{t}-D_{i}\left(a_{i j} D_{j} v\right)=0 & \text { in }\{v>0\}, \\ \frac{v_{t}}{|D v|}=F(x, D v):=a_{i j} D_{j} v \nu_{i} & \text { on } \partial\{v>0\}, \\ v(x, 0)=v_{0}(x) . & \end{cases}
$$

The unknown is the function $v(x, t)$, defined for $(x, t) \in \mathbb{R}^{n} \times[0, \infty)$ with values in $\mathbb{R}^{+}$. The vector $\nu=\left(\nu_{1}, \ldots, \nu_{n}\right)=\nu_{x, t}$ appearing in the second equation denotes the spatial normal vector at $(x, t) \in \partial\{v>0\}$, inward with respect to $\{v>0\}$.

The matrix $A(x)=\left(a_{i j}(x)\right)$ is assumed to be symmetric, bounded, and uniformly elliptic. In particular it satisfies

$$
\lambda|\xi|^{2} \leq \sum_{i, j} a_{i j}(x) \xi_{i} \xi_{j} \leq \Lambda|\xi|^{2} \quad \text { for all } x \in \mathbb{R}^{n} \text { and } \xi \in \mathbb{R}^{n}
$$

for some positive constants $\lambda$ and $\Lambda$. Throughout this paper, the initial data will be assumed to satisfy:

$$
v_{0} \in C^{0,1}\left(\left\{v_{0}>0\right\}\right) \text { and } \partial\left\{v_{0}>0\right\} \text { is of class } C^{1} .
$$

One-phase Stefan problems such as $(P)$ typically describe the melting of a frozen granular medium (at constant zero temperature) in contact with a liquified region. In this case, $v(x, t)$ denotes the temperature of the water and the coefficients $a_{i j}(x)$ describe the thermal diffusivity of the medium. The free boundary is the set

Received by the editors February 25, 2008.

2010 Mathematics Subject Classification. Primary 35Q35, 35Q80, 74Q10, 78M40.

The first author was partially supported by NSF-DMS 0700732 .

The second author was partially supported by NSF grant DMS-0456647.

(C)2010 American Mathematical Society Reverts to public domain 28 years from publication 
$\partial\{v(\cdot, t)>0\}$, which models the solid-liquid interface. The free boundary condition, which could also be written as

$$
v_{t}=F(x, D x)|D v|=a_{i j} D_{j} v D_{i} v
$$

says that the free boundary is moving with (outward) normal velocity $F(x, D v)$. Note that (1.1) yields

$$
\lambda|D v| \leq F(x, D v) \leq \Lambda|D v| \quad \text { on } \partial\{v>0\} .
$$

We refer to [P], R1] and [Rou] for further discussions about the model.

Even with smooth initial data, the existence of smooth solutions to the Stefan problem is not expected due to free boundary singularities such as merging of the fingers (or formation of holes in the ice cubes). The short time existence of classical solutions for $(P)$ was established by Hanzawa $[\mathrm{H}]$ when $v_{0}$ and $\partial\left\{v_{0}>0\right\}$ are of $\mathcal{C}^{\infty}$, and the existence of weak solutions in $H^{1}$ was proved by Kamenomostskaja Ka, Oleı̌nik [O] and Friedman [F] when $v_{0}$ satisfies (1.2). It was later observed by Duvaut $[\mathrm{Du}$ (see also Friedman and Kinderlehrer [FK] that if $v(x, t)$ is a smooth solution of $(P)$, then $u(x, t)=\int_{0}^{t} v(x, s) d s$ satisfies the following parabolic variational inequality:

$$
\left\{\begin{array}{l}
u(x, t) \geq 0, \\
\left(\partial_{t} u-D_{i}\left(a_{i j} D_{j} u\right)\right)(v-u) \geq f(v-u) \quad \text { a.e. }(x, t) \text { for any } v \geq 0, \\
u(x, 0)=0,
\end{array}\right.
$$

where

$$
f(x)= \begin{cases}v_{0}(x) & \text { in } \Omega_{0}, \\ -1 & \text { in } \mathbb{R}^{n} \backslash \Omega_{0} .\end{cases}
$$

This parabolic inequality always has a global unique solution $u(x, t)$ when $v_{0}$ satisfies (1.2). The corresponding time derivative $v=\partial_{t} u \in L^{2}\left(\mathbb{R}^{n} \times[0, \infty)\right)$ is thus sometimes called a weak solution of $(P)$. The regularity of $u$ and that of its free boundary have been studied by several authors. An important result, due to Caffarelli and Friedman $\left[\mathrm{CF}\right.$, says that when $a_{i j}=\delta_{i j}$ the temperature $\partial_{t} u$ is continuous in $\mathbb{R}^{n} \times[0, \infty)$. We refer to Rodrigues [R1] for a more detailed presentation of the weak formulations of $(P)$.

More recently, the notion of viscosity solution, which was first introduced by Crandall and Lions in CL for Hamilton-Jacobi equations, was developed in the framework of Hele-Shaw and Stefan problems by one of the authors K1 (see the next section for the precise definition). This notion of solution directly deals with the free boundary problem $(P)$ with point-wise, maximum-principle type arguments. The global existence and uniqueness of the viscosity solution is established in K1: here the uniqueness result holds with additional regularity assumptions on the initial data (mainly $v_{0} \in \mathcal{C}^{2}\left(\left\{v_{0}>0\right\}\right)$ and $\partial\left\{v_{0}>0\right\}$ in $\left.\mathcal{C}^{1,1}\right)$.

As we will see in this paper, each notion of solutions described above holds its own advantage for the analysis of $(P)$. Thus the natural question is whether weak and viscosity solutions coincide. Our first main result, Theorem 3.1, states that the answer is yes whenever the weak solution exists: i.e. when $v_{0}$ satisfies (1.2).

The second main result of the paper, which is also an application of the first, is to study the homogenization of the one-phase Stefan problem. Following Rodrigues 
[R2], we assume that the elliptic operator has fast oscillating coefficients: We define

$$
\mathcal{A}^{\varepsilon} v:=D_{i}\left(a_{i j}\left(\frac{x}{\varepsilon}\right) D_{j} v\right)
$$

where $A(y)=\left(a_{i j}\right)(y)$ is a symmetric matrix satisfying (1.1). We now consider the following Stefan problem with highly oscillating diffusion coefficients:

$\left(P^{\varepsilon}\right) \quad \begin{cases}\left(v^{\varepsilon}\right)_{t}-\mathcal{A}^{\varepsilon} v^{\varepsilon}=0 & \text { in }\left\{v^{\varepsilon}>0\right\}, \\ \frac{v_{t}^{\varepsilon}}{\left|D v^{\varepsilon}\right|}=F^{\varepsilon}\left(x, D v^{\varepsilon}\right):=a_{i j}\left(\frac{x}{\varepsilon}\right) D_{j} v^{\varepsilon} \nu_{i} & \text { on } \partial\left\{v^{\varepsilon}>0\right\}, \\ v^{\varepsilon}(x, 0)=v_{0}(x), & \end{cases}$

where $\nu=\left(\nu_{1}, \ldots, \nu_{n}\right)$ denotes the spatial normal vector to $\partial\left\{v^{\varepsilon}(\cdot, t)>0\right\}$. The object of Sections 4 and $[5$ is to investigate the behavior of (viscosity or weak) solutions $v^{\varepsilon}$ as $\varepsilon$ goes to 0 .

Interesting phenomena arise when we make some assumptions on the coefficients $a_{i j}(y)$ that guarantee some kind of averaging behavior. More precisely, besides (1.1), we also assume that $\left(a_{i j}(y)\right)$ satisfies:

(a) $a_{i j}(y)$ is a Lipschitz continuous function,

(b) $a_{i j}(y)$ has some averaging properties, i.e., one of the following holds:

(b1) $y \mapsto a_{i j}(y)$ is $\mathbb{Z}^{n}$-periodic,

(b2) $y \mapsto a_{i j}(y)$ is a stationary ergodic random variable over a probability space $(A, \mathcal{F}, P)$.

We recall that a random variable $g(x, \omega)$ is said to be stationary ergodic if it satisfies the following two conditions:

(1) The distribution of the random variable $g(x, \cdot): A \rightarrow \mathbb{R}$ is independent of $x$ (we say that $g$ is stationary). More precisely, we will assume that for every $x \in \mathbb{R}$ there exists a measure-preserving transformation $\tau_{x}: A \rightarrow A$ such that:

$$
g\left(x+x^{\prime}, \omega\right)=g\left(x, \tau_{x^{\prime}} \omega\right) \text { for all } x^{\prime} \in \mathbb{R}^{n} \text { and } \omega \in A .
$$

(2) The underlying transformation $\tau_{x}$ is ergodic, that is, if $B \subset A$ is such that $\tau_{x} B=B$ for all $x \in \mathbb{R}^{n}$, then $P(B)=0$ or 1.

Our main result states that under assumptions (a)-(b) and (1.2), the solution $\left(v^{\varepsilon}\right)$ of $\left(P^{\varepsilon}\right)$ converges locally uniformly (almost everywhere in the random case) to the unique solution $v(x, t)$ of

$$
\begin{cases}v_{t}-\mathcal{A}^{0} v=0 & \text { in }\{u>0\} \\ \frac{v_{t}}{|D v|}=F^{0}(D v):=q_{i j} D_{j} v \nu_{i} & \text { on } \partial\{v>0\} \\ v(x, 0)=v_{0}(x) & \end{cases}
$$

where $\left(q_{i j}\right)$ is a symmetric matrix with constant coefficients (see Section 3 for an explicit formula) and $\mathcal{A}^{0}$ is the corresponding elliptic operator:

$$
\mathcal{A}^{0} v:=D_{i}\left(q_{i j} D_{j} v\right) \text {. }
$$

A similar question was first addressed by J.-F. Rodrigues $\mathrm{R} 2$ in the case of periodic coefficients and for weak solutions of the Stefan problem, that is, for $u^{\varepsilon}(x, t)$, 
with bounded support, solving the variational inequality

$$
\left\{\begin{array}{l}
u^{\varepsilon}(x, t) \geq 0 \quad \text { in } \mathbb{R}^{n} \times[0, T] \\
\left(\partial_{t} u^{\varepsilon}-D_{i}\left(a_{i j}(x / \varepsilon) D_{j} u^{\varepsilon}\right)\right)\left(v-u^{\varepsilon}\right) \geq f\left(v-u^{\varepsilon}\right) \quad \text { a.e. }(x, t) \text { for any } v \geq 0 \\
u^{\varepsilon}(x, 0)=0
\end{array}\right.
$$

Here $f$ is defined by (1.3). The homogenization of variational inequalities as above is often simpler than that of free boundary problems such as $\left(P^{\varepsilon}\right)$. In Section 4 we give a generalized proof of Rodrigues' results that applies to the random coefficients case, using the notion of $\Gamma$-convergence and some results of $G$. Dal Maso and L. Modica DM1, DM2.

The difficulty is then to study the behavior of the free boundary itself as $\varepsilon$ goes to zero. Under the assumption that both $u^{\varepsilon}$ and $u$ have star-shaped free boundaries, it was shown in $\left[\mathrm{R} 2\right.$ that the oscillating free boundary $\partial\left\{u^{\varepsilon}>0\right\}$ converges in $L^{1}$ to the free boundary of the homogenized function $\partial\left\{u^{0}>0\right\}$. Unfortunately, the variational approach in $\mathrm{R} 2$ does not yield the uniform convergence of the free boundaries in the homogenization limit, except in some one-dimensional cases.

In Section 5, we improve the result of $\mathrm{R} 2$ by establishing the uniform convergence of the free boundaries and viscosity solutions for general initial data. Note that the positive phases of solutions for both problems $\left(P^{\varepsilon}\right)$ and $\left(P^{0}\right)$ may go through topological changes such as the merging of two fingers. Our result states that the oscillating free boundaries converge uniformly even in the event of such singularities.

The key point in the proof is the use of our first result, i.e., the fact that viscosity solutions of $\left(P^{\varepsilon}\right)$ (and $\left(P^{0}\right)$ ) are the "time derivatives" of the solution of the variational problem (Theorem [3.1). This enables us to combine the strong stability properties of the solutions of the obstacle problem with point-wise arguments available for viscosity solutions.

Corresponding results were obtained in $\mathrm{KM}$ for a quasi-static free boundary problem, i.e., with the heat operator $\partial_{t}-\mathcal{A}$ replaced by the Laplace operator $\Delta$ in $(P)$ (Hele-Shaw type problems). In that case, however, we strongly relied on the monotonicity of the solutions in time to establish the results. It turns out that the Stefan problem that we consider here is significantly more difficult to treat because of the lack of monotonicity and the presence of a time-dependent operator in the positive phase. We also point out that the elliptic operator $\left(\mathcal{A}^{\varepsilon}\right)$ in $\left(P^{\varepsilon}\right)$ is more general than the one considered in [KM] (which was the simple Laplace operator). Adapting some of the arguments presented here, it would naturally be possible to extend the results of $[\mathrm{KM}$ to more general elliptic operators.

The paper is organized as follows: In the next section, we recall the definition of weak solutions and viscosity solutions of the Stefan problem. In Section 3 , we prove our first main result, which states that both notions lead to the same solutions. We then (Sections 4 and 5) address the question of the homogenization of Stefan problems; i.e., we show the uniform convergence of the solutions of $\left(P^{\varepsilon}\right)$ to the solutions of $\left(P^{0}\right)$.

Notation. For any nonnegative function $w(x, t): \mathbb{R}^{n} \times[0, \infty) \rightarrow \mathbb{R}^{+}$, we will always denote

$$
\Omega(w)=\{w>0\}, \quad \Omega_{t}(w)=\{x ; w(x, t)>0\}
$$


and

$$
\Gamma(w)=\partial \Omega(w), \quad \Gamma_{t}(w)=\partial \Omega_{t}(w) .
$$

We call $\Omega_{t}(w)$ and $\Gamma_{t}(w)$, respectively, the positive phase and the free boundary of $w$.

\section{VARIATIONAL AND VISCOSity SOLUTIONS}

In this section, we denote by $A=\left(a_{i j}(x)\right)$ a symmetric matrix bounded and uniformly elliptic (i.e., satisfying (1.1)), and by $\mathcal{A}$ the corresponding uniformly elliptic operator:

$$
\mathcal{A} u:=D_{i}\left(a_{i j} D_{j} u\right)
$$

We now describe the notions of variational and viscosity solutions for the one-phase Stefan problem $(P)$.

2.1. Variational formulation. Let $v(x, t)$ be a classical solution of the Stefan problem $(P)$ in $\mathbb{R}^{n} \times[0, T]$ with initial condition

$$
v(x, 0)=v_{0}(x)
$$

Choose a bounded open set $\mathcal{O} \subset \mathbb{R}^{n}$ containing the support of $v_{0}$. Following $[\mathrm{FK}$ and $\mathrm{R} 2$ it can be shown that, if $\mathcal{O}$ is chosen large enough depending on $T$, then the function $u(x, t)=\int_{0}^{t} v(x, s) d s$ solves the following variational problem:

Problem 1. Find $u \in L^{2}\left(0, T ; H^{1}(\mathcal{O})\right)$ such that $u_{t} \in L^{2}(\mathcal{O} \times[0, T])$ and

$$
\left\{\begin{array}{l}
u(\cdot, t) \in \mathcal{K}(t), \\
\int_{\mathcal{O}} \partial_{t} u(v-u) d x+\int_{\mathcal{O}} a_{i j} D_{i} u D_{j}(v-u) d x \geq \int_{\mathcal{O}} f(v-u) d x \quad \forall v \in \mathcal{K}(t)
\end{array}\right.
$$

for a.e. $t \in(0, T)$ and satisfying $u(x, 0)=0$.

Here (and below), we set

$$
\mathcal{K}(t)=\left\{v \in H_{0}^{1}(\mathcal{O}) ; v \geq 0\right\}
$$

and

$$
f(x)= \begin{cases}v_{0}(x) & \text { for } x \in \Omega_{0}, \\ -1 & \text { for } x \notin \Omega_{0} .\end{cases}
$$

Furthermore, when the coefficients $a_{i j}(x)$ are Lipschitz continuous, this problem is equivalent to (see [FK]):

Problem 2. Find $u \in L^{2}\left(0, T ; H^{2}(\mathcal{O})\right)$ such that $u_{t} \in L^{2}(\mathcal{O} \times[0, T])$ and

$$
\left\{\begin{array}{l}
u(t) \in \mathcal{K}(t) \\
\left(\partial_{t} u-D_{j}\left(a_{i j} D_{i} u\right)\right)(v-u) \geq f(v-u) \quad \text { a.e. }(x, t) \in \mathcal{O} \times(0, T) \\
\text { for any } v \in \mathcal{K}(t) \\
u(x, 0)=0
\end{array}\right.
$$

The computations that lead to Problem 1 (and 2) can only be performed if $v$ is a classical solution of the Stefan problem. However, we have the following result:

Theorem 2.1 ([FK]). If $v_{0}$ satisfies (1.2), then Problem 1 (or 2) has a unique solution. 
We will thus say that if $u$ is a solution of Problem 1 (or 2), then $u_{t}$ is a weak (or variational) solution of the corresponding Stefan problem $(P)$. Weak solutions naturally agree with classical ones when they are regular enough.

Finally, let us notice that the solution $u(x, t)$ of Theorem 2.1 actually satisfies $u \in L^{\infty}\left(0, T ; W^{2, p}(\mathcal{O})\right)$ for $1 \leq p<\infty$ and $u_{t} \in L^{\infty}((0, T) \times \mathcal{O})$ and

$$
\begin{cases}u_{t}-\mathcal{A} u=v_{0}(x)-\chi_{\Omega_{t}(u)-\Omega_{0}(u),} & \\ u=|D u|=0 & \text { on } \Gamma(u), \\ u(x, 0)=0 . & \end{cases}
$$

Remark. The constraint set $\mathcal{O}$ in Problems 1 and 2 is introduced to make sure that the variational solution has bounded support. In fact, Lemma 3.6 in section 3 states that $u(x, t)$ is independent of the choice of $\mathcal{O}$ if $\mathcal{O}$ includes a sufficiently large ball.

2.2. Viscosity solution. Another way to define solutions of the Stefan problem is by viscosity solutions. In the context of Stefan and Hele-Shaw problems, viscosity solutions were first studied in [K1. We recall here the definitions and some important facts about those solutions.

For any nonnegative function $w(x, t)$, we define

$$
w_{*}(x, t):=\liminf _{(y, s) \rightarrow(x, t)} w(y, s)
$$

and

$$
w^{*}(x, t):=\limsup _{(y, s) \rightarrow(x, t)} w(y, s) .
$$

Letting $\Sigma \subset \mathbb{R}^{n} \times[0, \infty)$ be a space-time domain with smooth boundary, we recall the following definitions for viscosity subsolutions and supersolutions of $(P)$ (see K1]:

Definition 2.2. A nonnegative upper semicontinuous function $v(x, t)$ defined in $\Sigma$ is a viscosity subsolution of $(P)$ if the following hold:

(a) For all $T \in(0, \infty)$, the set $\overline{\Omega(v)} \cap\{t \leq T\} \cap \Sigma$ is bounded.

(b) For every $\phi \in C^{2,1}(\Sigma)$ such that $v-\phi$ has a local maximum in $\overline{\Omega(v)} \cap\{t \leq$ $\left.t_{0}\right\} \cap \Sigma$ at $\left(x_{0}, t_{0}\right)$, the following holds:

(i) If $v\left(x_{0}, t_{0}\right)>0$, then $\left(\phi_{t}-\mathcal{A} \phi\right)\left(x_{0}, t_{0}\right) \leq 0$.

(ii) If $\left(x_{0}, t_{0}\right) \in \Gamma(v),|D \phi|\left(x_{0}, t_{0}\right) \neq 0$ and $\left(\phi_{t}-\mathcal{A} \phi\right)\left(x_{0}, t_{0}\right)>0$, then

$$
\left(\phi_{t}-F(x, D \phi)|D \phi|\right)\left(x_{0}, t_{0}\right) \leq 0 .
$$

Definition 2.3. A nonnegative lower semicontinuous function $v$ defined in $\Sigma$ is a viscosity supersolution of $(P)$ if for every $\phi \in C^{2,1}(\Sigma)$ such that $v-\phi$ has a local minimum in $\Sigma \cap\left\{t \leq t_{0}\right\}$ at $\left(x_{0}, t_{0}\right)$, the following holds:

(i) If $v\left(x_{0}, t_{0}\right)>0$, then $\left(\phi_{t}-\mathcal{A} \phi\right)\left(x_{0}, t_{0}\right) \geq 0$.

(ii) If $\left(x_{0}, t_{0}\right) \in \Gamma(v),|D \phi|\left(x_{0}, t_{0}\right) \neq 0$ and $\left(\phi_{t}-\mathcal{A} \phi\right)\left(x_{0}, t_{0}\right)<0$, then

$$
\left(\phi_{t}-F(x, D \phi)|D \phi|\right)\left(x_{0}, t_{0}\right) \geq 0 .
$$

Now let $v_{0}(x)$ be a given initial condition with support $\Omega_{0}$ and free boundary $\Gamma_{0}=\partial \Omega_{0}$ and let $Q=\mathbb{R}^{n} \times(0, \infty)$. Then we have the following definitions. 
Definition 2.4. The function $v(x, t)$ is a viscosity subsolution of $(P)$ in $Q$ with initial data $v_{0}$ if

(a) $v$ is a viscosity subsolution of $(P)$ in $Q$,

(b) $v$ is upper semicontinuous in $\bar{Q}$ and $v(x, 0)=v_{0}(x)$,

(c) $\overline{\Omega(v)} \cap\{t=0\}=\overline{\Omega\left(v_{0}\right)}$.

Definition 2.5. The function $v(x, t)$ is a viscosity supersolution of $(P)$ in $Q$ with initial data $v_{0}$ if

(a) $v$ is a viscosity supersolution in $Q$,

(b) $v$ is lower semicontinuous in $\bar{Q}$ and $v(x, 0)=v_{0}(x)$.

A viscosity subsolution $v$ of $(P)$ is upper semi-continuous by definition, and thus can be positive on $\Gamma(u)$. However, the following lemma, to be used in Section 3, states that $u$ cannot have an isolated jump.

Definition 2.6. The function $v(x, t)$ is a viscosity solution of $(P)$ (in $Q$ with initial data $v_{0}$ ) if $v$ is a viscosity supersolution and $v^{*}$ is a viscosity subsolution of $(P)$ (in $Q$ with initial data $\left.v_{0}\right)$.

The existence of viscosity solutions and their properties have been studied in great detail in [K1]. In particular, we have:

Theorem 2.7. Assume that the initial data $v_{0}(x)$ satisfies

$$
v_{0} \in \mathcal{C}^{2}\left(\Omega_{0}\right), \Gamma_{0}=\partial \Omega_{0} \text { in } \mathcal{C}^{1,1} \text { and } \mathcal{A} v_{0}>0 \text { on } \Gamma_{0} .
$$

Then $(P)$ admits a unique viscosity solution defined for all time $t \geq 0$.

A more general comparison principle will be shown later (Corollary 3.12) once we prove the coincidence of viscosity solutions with the weak solution (Theorem 3.1). In this paper, one of the most important features of viscosity solutions is the fact that they satisfy a comparison principle:

We say that a pair of functions $u_{0}, v_{0}: \bar{D} \rightarrow[0, \infty)$ are (strictly) separated (denoted by $u_{0} \prec v_{0}$ ) in $D \subset \mathbb{R}^{n}$ if

(i) $\overline{\left\{u_{0}>0\right\}} \cap \bar{D}$ is compact and

(ii) $u_{0}(x)<v_{0}(x)$ in $\overline{\left\{u_{0}>0\right\}} \cap \bar{D}$.

Then we have the following theorem:

Theorem 2.8 (Comparison principle). Let $v_{1}, v_{2}$ be respectively viscosity subsolutions and supersolutions of $(P)$ in $\Sigma$. If $v_{1} \prec v_{2}$ on the parabolic boundary of $\Sigma$, then $v_{1}(\cdot, t) \prec v_{2}(\cdot, t)$ in $\Sigma$.

Sketch of the proof. The proof is parallel to that of Theorem 1.7 in K1. The difference from the original proof in [K1, which deals with the Hele-Shaw flow, is twofold. On the one hand, one has to deal with the dependence of the free boundary velocity on $x$ and $\nu$. A modified proof to deal with this is presented in $\mathrm{K2}$. On the other hand, one also has to construct smooth, local barriers which solve the equation

$$
h_{t}-\mathcal{A} h \leq 0 \quad(\text { or } \geq 0)
$$

in its support, with $|D h|>0$ on $\partial\{h>0\}$. We briefly outline how to construct such barriers in Appendix $\mathrm{A}$.

Note that the proof in [K1] uses strongly the fact that the initial data are strictly separated. In section 3 we will show that the comparison principle actually holds without strict separation of the initial data (see Corollary 3.12). 


\section{UNITING NOTIONS OF WEAK AND VISCOSITY SOLUTIONS}

Now we establish the fact that variational solutions (given by Theorem 2.1) are indeed viscosity solutions of the Stefan problem $(P)$. More precisely, we prove:

Theorem 3.1. Assume that $v_{0}$ satisfies (1.2). Let $u(x, t)$ be the unique solution of (2.1) in $\mathcal{O} \times[0, T]$ and let $v(x, t)$ be the solution of

$$
\begin{cases}v_{t}-\mathcal{A} v=0 & \text { in } \Omega(u), \\ v=0 & \text { on } \Gamma(u),\end{cases}
$$

with initial data

$$
v(x, 0)=v_{0}(x)
$$

Then the following hold:

(i) $v(x, t)$ is a viscosity solution of $(P)$ in $\mathcal{O} \times[0, T]$ with initial data $v(x, 0)=$ $v_{0}(x)$.

(ii) $u(x, t)=\int_{0}^{t} v(x, s) d s$.

Before presenting the proof of Theorem 3.1, it is necessary to recall the main properties of $u$.

3.1. Properties of the variational solutions. The existence of a unique solution to (2.1) is given by Theorem 2.1 (see [FK], R2]). Furthermore, we have

$$
\begin{gathered}
u \in L^{\infty}\left(0, T, H^{2, p}(\mathcal{O})\right) \quad \text { for } 1 \leq p<\infty, \\
u_{t} \in L^{\infty}(\mathcal{O} \times(0, T)),
\end{gathered}
$$

and $u$ satisfies

$$
\left\{\begin{array}{l}
u_{t}-\mathcal{A} u \geq f, \quad u \geq 0, \\
u\left(u_{t}-\mathcal{A} u-f\right)=0 \quad \text { a.e. in } \mathcal{O} \times(0, \infty) .
\end{array}\right.
$$

With minor adaptations from [FK] (see also [R2]), we can also state:

Proposition 3.2. The unique solution $u$ of (2.1) satisfies

$$
0 \leq u_{t} \leq C \quad \text { a.e. } \mathcal{O} \times(0, T),
$$

where $C$ is a constant depending on $f, \lambda$ and $\Lambda$. In particular, $u$ is Lipschitz with respect to $t$ and $u$ is $\mathcal{C}^{\alpha}$ with respect to $x$ for all $\alpha \in(0,1)$. Furthermore if $0 \leq t<s \leq T$, then $u$ satisfies $u(\cdot, t)<u(\cdot, s)$ and so:

$$
\Omega_{0} \subset \Omega_{s}(u) \subset \Omega_{t}(u)
$$

Lemma 3.3. Suppose $x_{0} \in \overline{\Omega_{t_{0}}(u)}$ and assume that $B_{r}\left(x_{0}\right) \cap \Omega_{0}=\emptyset$ for some $r$. Then there exists a constant $C$, depending only on $n, \lambda$ and $\Lambda$, such that

$$
\sup _{x \in B_{r}\left(x_{0}\right)} u\left(x, t_{0}\right)>C r^{2} \text {. }
$$

Proof. The proof is classical and makes use of the barrier $h(x)$ constructed in Appendix A] We first assume that $x_{0} \in \Omega_{t_{0}}(u)$ (the result then follows by continuity of $u)$. Let $Q_{r}\left(x_{0}, t_{0}\right)=B_{r}\left(x_{0}\right) \times\left[t_{0}-r^{2}, t_{0}\right]$ and define

$$
w(x, t)=u(x, t)-\frac{1}{2 n} h\left(x-x_{0}\right)+\frac{1}{2}\left(t-t_{0}\right) .
$$


It is readily seen that $\{w>0\} \cap Q_{r}\left(x_{0}, t_{0}\right) \subset\{u>0\} \cap Q_{r}\left(x_{0}, t_{0}\right)$ and since $\partial_{t} u-\mathcal{A} u=-1$ in $\{u>0\} \backslash \Omega_{0}$, we have

$$
\partial_{t} w-\mathcal{A} w=0 \quad \text { in }\{w>0\} \cap Q_{r}\left(x_{0}, t_{0}\right) .
$$

Since $w\left(x_{0}, t_{0}\right)>0$, the maximum of $w$ in $Q_{r}\left(x_{0}, t_{0}\right)$ is nonnegative and is thus reached in $\{w>0\} \cap \partial_{p} Q_{r}\left(x_{0}, t_{0}\right)$ ( $\partial_{p}$ denotes the parabolic boundary).

Using the quadratic growth of $h$, we easily deduce that

$$
\sup _{Q_{r}\left(x_{0}, t_{0}\right)} u(x, t) \geq C r^{2},
$$

and the fact that $u$ is nondecreasing with respect to $t$ yields the result.

Lemma 3.4. Let $0 \leq s<t$. Then $\Omega_{t}(u)$ lies within a $C(t-s)^{1 / 2}$-neighborhood of $\Omega_{s}(u)$ :

In particular

$$
\Omega_{t}(u) \subset \Omega_{s}(u)+B_{C(t-s)^{1 / 2}} .
$$

$$
\Omega_{t}(u) \subset \Omega_{0}+B_{C t^{1 / 2}} \quad \text { for all } t>0
$$

Proof. Let $x_{0}$ be a point in $\Omega_{t}(u)$ at a distance $\delta$ of $\Omega_{s}(u)$. Then $B_{\delta}\left(x_{0}\right) \cap \Omega_{0}=\emptyset$, so Lemma 3.3 implies that

$$
\sup _{B_{\delta}\left(x_{0}\right)} u(\cdot, t) \geq C \delta^{2} .
$$

Since $u(x, t)-u(x, s) \leq C(t-s)$ for all $x$ ( $u$ is Lipschitz in time by Proposition 3.2), and $u(\cdot, s)=0$ in $B_{\delta}\left(x_{0}\right)$, we deduce that $\delta \leq C(t-s)^{1 / 2}$, which yields the result.

Lemma 3.5 (Comparison principle). Let $\Sigma$ be a smooth domain in $\mathbb{R}^{n} \times[0, \infty)$. Suppose that $u_{1}(x, t)$ and $u_{2}(x, t)$ solve (2.1) with $v_{0}^{1}(x)$ and $v_{0}^{2}(x)$ replacing $v_{0}(x)$. If $v_{0}^{1} \leq v_{0}^{2}$ in $\Sigma$ and $u_{1} \leq u_{2}$ in the parabolic boundary of $\Sigma$, then $u_{1} \leq u_{2}$ in $\Sigma$.

Proof. Let $w=u_{2}-u_{1}$ and assume that $w(x, t)$ has a negative minimum at $\left(x_{0}, t_{0}\right)$. Since $w \geq 0$ on the parabolic boundary of $\Sigma$, we have $\left(x_{0}, t_{0}\right) \notin \partial_{p} \Sigma$.

Moreover $0 \leq u_{2}\left(x_{0}, t_{0}\right)<u_{1}\left(x_{0}, t_{0}\right)$ and so $\partial_{t} u_{1}-\mathcal{A} u_{1}=f_{1}$ at $\left(x_{0}, t_{0}\right)$. We deduce

$$
\partial_{t} w-\mathcal{A} w \geq f_{2}-f_{1} \geq 0 \quad \text { at }\left(x_{0}, t_{0}\right),
$$

and the strong maximum principle for parabolic equations gives a contradiction.

The next lemma claims that the support of $u(\cdot, t)$ remains bounded at all times:

Lemma 3.6. For all $T>0$, there exists $M=M(T)$ depending only on $T$ and the initial data such that the solution $u(x, t)$ of (2.1) in $\mathcal{O} \times[0, T]$ satisfies

$$
\{u(\cdot, t)>0\} \subset B_{M}(0) \quad \text { for all } 0 \leq t \leq T
$$

(as long as $B_{M}(0) \subset \mathcal{O}$ ).

Proof. See Appendix A for the proof.

In particular, the above lemma says that if $\mathcal{O}$ is big enough, the choice of $\mathcal{O}$ is irrelevant in our problem.

Lastly we will need the following particular case of a stability result for a parabolic variational inequality. 
Lemma 3.7. If $v_{0}^{n}(x)$ and its support converge to $v_{0}(x)$ and $\Omega_{0}$ in $L^{n+1}$, then the solutions $u_{n}(x, t)$ of (2.1) with $v_{0}^{n}$ instead of $v_{0}$ converge uniformly to the solution $u(x, t)$ of (2.1).

Proof. It follows easily from the stability result in Appendix B.

3.2. Proof of Theorem [3.1, We are now ready to prove our main result. But first, $v$ needs a precise definition.

Since $u$ is continuous, and thanks to Lemma 3.6, the domain $\Omega(u)$ is a bounded open set of $\mathbb{R}^{n} \times \mathbb{R}^{+}$. The existence of a solution to (3.1) when $\Omega(u)$ is not smooth is then provided by Perron's method as follows (see GL]):

$$
v=\sup \left\{w \mid w_{t}-\mathcal{A} w \leq 0 \text { in } \Omega(u), w \leq 0 \text { on } \Gamma(u), w(x, 0) \leq v_{0}(x)\right\} .
$$

Classical potential theory assures that $v$ is continuous in $\Omega(u)$ and that $v_{t}-\mathcal{A} v=0$ in the classical sense. Note that it is not true in general that $v$ attains continuously its boundary value; in particular, it may happen that $\lim \sup _{(x, t) \rightarrow\left(x_{0}, t_{0}\right)} v(x, t)>0$ for some $\left(x_{0}, t_{0}\right) \in \Gamma(u)$.

We also should check that $v$ satisfies the initial condition. This actually follows rather easily from Lemma 3.4

Lemma 3.8. The function $v(x, t)$ satisfies the following initial condition:

$$
v(x, 0)=v^{*}(x, 0)=v_{0}(x) \quad \text { for all } x \in \mathbb{R}^{n}
$$

and

$$
\overline{\{u>0\}} \cap\{t=0\}=\overline{\Omega_{0}} .
$$

Proof.

1. Let $w(x, t)$ be the classical solution of $w_{t}-\mathcal{A} w=0$ in $\Omega_{0} \times(0, \infty)$ with $w(x, t)=0$ for $x \in \partial \Omega_{0}$ and $w(x, 0)=v_{0}(x)$. Then we have $v(x, t) \geq w(x, t)$ in $\Omega_{0} \times(0, \infty)$ and so

$$
\lim _{s \rightarrow 0} v(x, s) \geq v_{0}(x)
$$

hence $v^{*}(x, 0) \geq v(x, 0) \geq 0$.

2. Now let $w_{\tau}$ denote the classical solution of $\left(\partial_{t}-\mathcal{A}\right) w=0$ in $\left(\Omega_{0}+B_{C \tau^{1 / 2}}\right) \times(0, \tau)$ which vanishes on $\partial\left(\Omega_{0}+B_{C \tau^{1 / 2}}\right)$ and such that $w(x, 0)=v_{0}(x)$. Then Lemma 3.4 implies that

$$
v(x, t) \leq w_{\tau}(x, t) \quad \text { for all } t \leq \tau .
$$

This gives

$$
\lim _{s \rightarrow 0^{+}} v(x, s) \leq w_{\tau}(x, 0),
$$

and it is readily seen that $w_{\tau}(x, 0)=v_{0}(x)$. It follows that

$$
\lim _{s \rightarrow 0^{+}} v(x, s) \leq v_{0}(x),
$$

and the continuity of $v_{0}$ yields

$$
v(x, 0) \leq v^{*}(x, 0) \leq v_{0}(x) .
$$

3. Finally, Proposition 3.2 gives $\Omega_{0} \subset \Omega_{t}(u)$ for all $t>0$ and so

$$
\overline{\Omega_{0}} \subset \overline{\{u>0\}} \cap\{t=0\},
$$

and the last equality in Lemma 3.8 follows from Lemma 3.4 
In the sequel, we extend $v(x, t)$ by 0 outside $\Omega(u)$. Since the function $w \equiv 0$ satisfies all the conditions in (3.5), we must have $v(x, t) \geq 0$ in $\mathbb{R}^{n} \times \mathbb{R}^{+}$, so $v(x, t)$ is lower semicontinuous in $\mathbb{R}^{n} \times \mathbb{R}^{+}$(recall that it may happen that $\lim \sup _{(x, t) \rightarrow\left(x_{0}, t_{0}\right)} v(x, t)$ $>0$ for some $\left.\left(x_{0}, t_{0}\right) \in \Gamma(u)\right)$. Moreover, we have:

Lemma 3.9. The solution $v$ of (3.1) is strictly positive in $\Omega(u)$ and satisfies

$$
\{v>0\}=\Omega(u) \quad \text { and } \quad \Omega(u) \subset\left\{v^{*}>0\right\} \subset \bar{\Omega}(u) .
$$

In particular, $v$ satisfies $\partial\{v>0\}=\partial\left\{v^{*}>0\right\}=\Gamma(u)$.

Proof. Assume that $v\left(x_{0}, t_{0}\right)=0$ for some $\left(x_{0}, t_{0}\right) \in \Omega(u)$. Then, the strong maximum principle implies that $v=0$ in the set $S\left(x_{0}, t_{0}\right)$ of all the points in $\Omega(u)$ which can be connected to $\left(x_{0}, t_{0}\right)$ by a polygonal line contained in $\Omega(u)$ along which $t$ is increasing.

The claim is then

$$
\left(\overline{S\left(x_{0}, t_{0}\right)} \cap\{t=0\}\right) \cap \Omega_{0} \neq \emptyset,
$$

which leads to a contradiction thanks to (3.6).

To prove (3.7), note that if it does not hold, then $\overline{S\left(x_{0}, t_{0}\right)} \cap\left(\Omega_{0} \times\left(0, t_{0}\right)\right)=\emptyset$ (for if $\left(x_{1}, t_{1}\right) \in S$ with $x_{1} \in \Omega_{0} \subset \Omega_{t}(u)$, then $\left(x_{1}, t\right) \in S$ for all $\left.t \in\left(0, t_{1}\right)\right)$. Therefore it follows that

$$
\left(\partial_{t}-\mathcal{A}\right) u \leq 0 \quad \text { in } S\left(x_{0}, t_{0}\right) .
$$

Furthermore, $\partial S\left(x_{0}, t_{0}\right) \subset\{u=0\}$ and so $u=0$ on $\partial S\left(x_{0}, t_{0}\right)$. The weak maximum principle (applied to $u$ ) thus yields $u=0$ in $S\left(x_{0}, t_{0}\right)$, which contradicts the fact that $\left(x_{0}, t_{0}\right) \in\{u>0\}$.

The next lemma allows us to approximate the initial data $v_{0}(x)$ in such a way that the approximating solution satisfies $\mathcal{A} v_{0}^{n}>0$ near the initial free boundary.

Lemma 3.10. Suppose $v_{0}$ satisfies (1.2). Then there exists a monotone decreasing sequence $v_{0}^{n}(x)$ such that

(a) $v_{0} \prec v_{0}^{n}$ in $\mathbb{R}^{n}$,

(b) $v_{0}^{n}$ and its support converge to $v_{0}$ and its support,

(c) $v_{0}^{n}$ satisfies $\mathcal{A} v_{0}^{n}>0$ near $\Gamma\left(v_{0}^{n}\right)$ and the corresponding solutions $v^{n}(x, t)$ of (3.1) satisfy:

$$
v_{0}^{n}(x) \leq\left(1+\delta\left(h_{0}\right)\right) v^{n}(x, t) \quad \text { for } 0 \leq t \leq h_{0},
$$

where $\delta\left(h_{0}\right)$ and $h_{0}$ depend on $n$ and satisfy $\delta\left(h_{0}\right) \rightarrow 0$ as $h_{0} \rightarrow 0$.

Similarly, there exists a sequence $\underline{v}_{0}^{n}$ such that $\underline{v}_{0}^{n} \prec v_{0}$ and $\underline{v}_{0}^{n}$ satisfies (b) and (c) above.

Proof.

1. Define

$$
\Omega_{0}^{n}=\left\{y: d\left(y, \Omega_{0}\right)<\frac{1}{n}\right\} .
$$

Let $K$ be a small ball $K \subset \Omega_{0}$, and let $c_{0}$ be a small positive number. Let $w_{0}^{n}(x, t)$ solve

$$
\begin{cases}\mathcal{A} w_{0}^{n}=c_{0} & \text { in } \Omega_{0}^{n}-K, \\ w_{0}^{n}=\min \left(\frac{1}{n}, \frac{1}{2} v_{0}\right) & \text { on } \partial K, \\ w_{0}^{n}=0 & \text { on } \partial \Omega_{0}^{n} .\end{cases}
$$


A solution to this problem exists and is strictly positive if $c_{0}$ is sufficiently small $\left(c_{0}\right.$ depends on $n$ ).

Then $v_{0}^{n}:=\max \left(w_{0}^{n},\left(1+\frac{1}{n}\right) v_{0}\right)$ is a continuous function with support $\Omega_{0}^{n}$ and such that $v_{0}^{n}=w_{0}^{n}$ near the boundary of $\Omega_{0}^{n}$. It is readily seen that (a) and (b) hold.

Now let $u^{n}$ solve (2.1) with $v_{0}^{n}$ instead of $v_{0}$, and let $v^{n}$ be the corresponding solution of (3.1) (with $u^{n}$ and $v_{0}^{n}$ replacing $u$ and $v_{0}$ ). The function $v^{n}(x, t)$ is greater than the solution $w^{n}(x, t)$ of the following boundary problem:

$$
\begin{cases}\left(\partial_{t}-\mathcal{A}\right) w^{n}=0 & \text { in } \Omega_{0}^{n} \times[0, \infty), \\ w^{n}(x, t)=0 & \text { for } x \in \partial \Omega_{0}^{n}, \\ w^{n}(x, 0)=v_{0}^{n}(x) & \text { for } x \in \Omega_{0}^{n} .\end{cases}
$$

Hence in order to prove that (c) holds, it is enough to prove that for $0 \leq t \leq h_{0}$ we have

$$
v_{0}^{n}(x) \leq\left(1+\delta\left(h_{0}\right)\right) w^{n}(x, t), \quad \text { with } \delta\left(h_{0}\right) \rightarrow 0 \text { as } h_{0} \rightarrow 0 .
$$

This inequality follows easily after noticing that in a small $\mathcal{O}\left(\frac{1}{n}\right)$-neighborhood of $\partial \Omega_{0}^{n}$, we have $\mathcal{A} w^{n}(x, 0)=c_{0}>0$ and so the function $w^{n}(x, t)$ is increasing with respect to $t$ for small $t$. Away from $\partial \Omega_{0}^{n}$, the function $w^{n}$ is strictly positive and continuous in time, so the inequality holds.

2. To prove the existence of $\underline{v}_{0}^{n}$, consider an increasing sequence of domain $\widetilde{\Omega}_{0}^{n}$ slightly smaller than $\Omega_{0}$ (for example, consider

$$
\left.\widetilde{\Omega}_{0}^{n}=\left\{y \in \Omega_{0}: d\left(y, \Gamma_{0}\right)>\frac{1}{n}\right\}\right) .
$$

Define $\underline{v}_{0}^{n}$ to be a sequence of continuous functions supported in $\widetilde{\Omega}_{0}^{n}$ with $\underline{v}_{0}^{n} \prec v_{0}$ and uniformly converging to $v_{0}$; for example, one can take $\underline{v}_{0}^{n}=\left(1-\frac{1}{n}\right) v_{0}$ in $\widetilde{\Omega}_{0}^{n / 2}$ and let $\underline{v}_{0}^{n}$ be the solution of $\mathcal{A} w=c_{0}>0$ in $\widetilde{\Omega}_{0}^{n}-\widetilde{\Omega}_{0}^{n / 2}$, with appropriate boundary data.

Proceeding as in Step 1, it is now straightforward to check that (c) holds.

We now proceed with the proof of Theorem 3.1

Proof of Theorem 3.1. Recall that the function $v(x, t)$ is lower semicontinuous and vanishes on $\Gamma(u)$. It satisfies $\Omega(v)=\Omega(u)$ and $\Omega(u) \subset \Omega\left(v^{*}\right) \subset \bar{\Omega}(u)$ (note that $v^{*}$ may be positive on $\Gamma(u)$ if the free boundary has a sharp cusp), and it solves

$$
v_{t}-\mathcal{A} v=0 \quad \text { in } \Omega(u) .
$$

Moreover, it is readily seen from (3.5) that $v$ is a supremum of subsolutions of $w_{t}-\mathcal{A} w=0$ in $\mathbb{R}^{n} \times(0, T)$ (take $\max (0, w)$ instead of $w$ in (3.5)). We thus have:

$$
v_{t}-\mathcal{A} v \leq 0 \quad \text { and } v_{t}^{*}-\mathcal{A} v^{*} \leq 0 \quad \text { in } \mathbb{R}^{n} \times(0, T) .
$$

The claim is that $v^{*}$ is a viscosity subsolution of $(P)$. Similar arguments would yield that $v$ is a supersolution of $(P)$, thus showing the first part of Theorem 3.1 .

The proof of the claim comes in two steps:

- First, we assume that $v_{0}(x)$ is such that for some positive constant $h_{0}$ and for $0 \leq t \leq h_{0}$ we have

(3.8) $\left\{\begin{array}{l}\mathcal{A} v_{0}>0 \text { in a neighborhood of } \Gamma\left(v_{0}\right), \\ v_{0}(x) \leq\left(1+\delta\left(h_{0}\right)\right) v(x, t) \text { for } t \leq h_{0}, \text { with } \delta\left(h_{0}\right) \rightarrow 0 \text { as } h_{0} \rightarrow 0 .\end{array}\right.$ 
This condition implies that $v$ is "almost monotone" near the free boundary. This will enable the comparison between the finite time difference of $u$ and $v$ (recall that the eventual goal is to prove $v=u_{t}$ ). This is crucial in the proof of (3.10) below.

- In the second part of the proof, Lemma 3.10 will be used to show that the result holds without condition (3.8).

Step 1 (When $v_{0}$ satisfies (3.8)). 1. First note that (3.8) implies that for any $h_{0}$, there exists $\delta$ such that

$$
\left(\partial_{t}-\mathcal{A}\right)((1+\delta) t v(x, t+\tau))=(1+\delta) v(x, t+\tau) \geq v_{0}
$$

in $\Omega(u) \cap\left\{0 \leq t+\tau \leq h_{0}\right\}$. The classical comparison principle applied to $u(x, t)$ and $(1+\delta) t v(x, t+\tau)$ in $\Omega(u) \cap\{0 \leq t \leq h\}$ thus yields:

$$
u(x, h) \leq(1+\delta) h v(x, h+\tau) \quad \text { for } 0 \leq \tau \leq h_{0}-h .
$$

We now claim that for $0<h<h_{0}, t \geq h$ and $0 \leq \tau<h_{0}-h$, the following holds:

$$
u_{h}^{-}(x, t):=\frac{u(x, t)-u(x, t-h)}{h} \leq(1+\delta) v(x, t+\tau)
$$

(recall that $\delta>0$ and $\delta \rightarrow 0$ as $h_{0} \rightarrow 0$ ).

When $t=h$, (3.10) follows from (3.9) (and the fact that $u(x, 0)=0$ ). When $t>h$ it follows from the definition of $v$ and the maximum principle, since $u_{h}^{-}$is a smooth function satisfying

$$
\left(\partial_{t}-\mathcal{A}\right)\left(u_{h}^{-}\right)=\frac{-1}{h} \chi_{\Omega_{t}(u) \backslash \Omega_{t-h}(u)} \leq 0
$$

with support in $\Omega(u)$ and $u_{h}^{-}=0$ on $\Gamma(u)$.

2. Let $\Sigma$ be a parabolic neighborhood of $\left(x_{0}, t_{0}\right)$ and assume that there is a $C^{2,1}$ function $\phi(x, t)$ such that $v^{*}-\phi$ has a local maximum zero at $\left(x_{0}, t_{0}\right) \in \bar{\Omega}(v)$ with $t_{0}>0$ in $\Sigma \cap \bar{\Omega}(v)$. We are going to show that $\phi$ satisfies the conditions (i) or (ii) in Definition 2.2

If $\left(x_{0}, t_{0}\right) \in \Omega(v)$, then $v_{t}^{*}-\mathcal{A} v^{*} \leq 0$ and so $\left(\phi_{t}-\mathcal{A} \phi\right)\left(x_{0}, t_{0}\right) \leq 0$. If $\left(x_{0}, t_{0}\right) \in$ $\Gamma(v)$ and $v^{*}\left(x_{0}, t_{0}\right)>0$, then $\phi$ is strictly positive in a neighborhood of $\left(x_{0}, t_{0}\right)$, and thus $v^{*}-\phi$ has a local maximum in a neighborhood of $\left(x_{0}, t_{0}\right)$ (note that the maximum is not just in $\overline{\Omega(v)})$. Since $v_{t}^{*}-\mathcal{A} v^{*} \leq 0$ in $\mathbb{R}^{n} \times(0, T)$, it follows that $\left(\phi_{t}-\mathcal{A} \phi\right)\left(x_{0}, t_{0}\right) \leq 0$.

Thus it remains to check that if $\left(x_{0}, t_{0}\right) \in \Gamma(v)$ with $v^{*}\left(x_{0}, t_{0}\right)=\phi\left(x_{0}, t_{0}\right)=0$ and

$$
|D \phi|\left(x_{0}, t_{0}\right) \neq 0, \quad\left(\phi_{t}-\mathcal{A} \phi\right)\left(x_{0}, t_{0}\right)>0,
$$

then (2.2) holds.

3. Suppose that

$$
\left[\phi_{t}-F(x, D \phi)|D \phi|\right]\left(x_{0}, t_{0}\right)>0 .
$$

We define $\Sigma_{1}:=B_{r}\left(x_{0}, t_{0}\right) \times\left[t_{0}-r, t_{0}\right]$ with $r$ small enough so that $\Sigma_{1} \subset \Sigma$ and $\Sigma_{1} \cap \Omega_{0}=\emptyset$. The goal is to construct a radially symmetric (in space) smooth function $\varphi(x, t)$ in $\Sigma_{1}$, which satisfies

(a) $\left(1+\delta_{0}\right) v^{*} \prec \varphi$ on the parabolic boundary of $\Sigma_{1}$ (for a small $\delta_{0}>0$ ),

(b) $|D \varphi|>0$ and $\varphi_{t}-\mathcal{A} \varphi>0$ in $\Sigma_{1} \cap\{\varphi>0\}$,

(c) $\varphi_{t}-F(x, D \varphi)|D \varphi|>0$ in $\Sigma_{1} \cap \bar{\Omega}(v)$,

(d) $\left(x_{0}, t_{0}\right) \in \Gamma(\varphi)$. 
The construction of such a function $\varphi$ is based on a Taylor expansion of $\phi$ near $\left(x_{0}, t_{0}\right)$. For more details, refer to $[\mathrm{KM}$, where a similar argument can be found in the proof of Theorem 3.1.

Note that $\Omega(v)=\Omega(u)$ does not jump in time (Lemma 3.4), and so it is possible to perturb $\varphi$ such that (a)-(c) hold together with

$$
\left(\mathrm{d}^{\prime}\right) \quad\left(x_{0}, t_{0}\right) \in \operatorname{Int}\{\varphi=0\}
$$

i.e., $\Omega\left(v^{*}\right)$ has crossed $\Omega(\varphi)$ from below in $\Sigma_{1}$ before $t=t_{0}$.

4. Fix $h>0$ such that $h \ll r$ and $h \ll h_{0}$. We introduce the function

$$
w(x, t)=\int_{t-h}^{t} \varphi(x, s) d s
$$

defined in $\Sigma_{1}$. Conditions (b) and (c) above guarantee that $\varphi$ is a supersolution of the Stefan problem $(P)$ in $\Sigma_{1}$, and since $\varphi$ is smooth, the classical computation gives that $w$ is a supersolution of the corresponding variational inequality. More precisely $w$ satisfies

$$
\left(w_{t}-\mathcal{A} w\right)(\cdot, t) \geq-\chi_{\Omega_{t}(w)-\Omega_{t-h}(w)}
$$

in $\Sigma_{1}$ (see Section 2.1).

Let $\tilde{u}(x, t)=u(x, t-h)-u(x, t-2 h)$ and let $\delta_{0}$ be the small constant in the construction of $\varphi$. Due to (3.10) for $t \in\left[t_{0}-r, t_{0}\right]$ the function $\tilde{u}$ satisfies

$$
\tilde{u}(x, t) \leq\left(1+\delta_{0}\right) h v(x, \tau) \quad \text { for } \tau \in[t-h, t]
$$

provided $0<h \leq h_{0} / 2$ (with $h_{0}$ small enough). Consequently

$$
\tilde{u}<w \quad \text { on } \partial B_{r}\left(x_{0}\right) \times\left[t_{0}-r, t_{0}\right] .
$$

Proposition 3.2 yields $\Omega_{t}(\tilde{u})=\Omega_{t-h}(u)$ and so $\tilde{u}$ satisfies, in $\Sigma_{1}$,

$$
\begin{aligned}
\tilde{u}_{t}-\mathcal{A} \tilde{u} & =-\chi_{\Omega_{t-h}(u)-\Omega_{t-2 h}(u)} \\
& =-\chi_{\Omega_{t}(\tilde{u})-\Omega_{t-h}(\tilde{u})} .
\end{aligned}
$$

Hence the comparison principle for the obstacle problem (Lemma 3.5) gives $\tilde{u} \leq w$ in $\Sigma_{1}$. In particular

$$
\Omega_{t-h}(u)=\Omega_{t}(\tilde{u}) \subset \Omega_{t}(w)=\Omega_{t}(\varphi) \text { in } B_{r}\left(x_{0}\right)
$$

for $t_{0}-r \leq t \leq t_{0}$. Since $h$ is arbitrary, this contradicts the fact that $\Omega(v)$ crosses $\Omega(\varphi)$ in $\Sigma_{1}$.

This completes the proof of the fact that $v^{*}$ is a subsolution of $(P)$. A similar argument would prove that $v$ is a supersolution of $(P)$, thus showing the first part of Theorem 3.1 when condition (3.8) holds.

5. Now we prove that (ii) holds: the time integral of the viscosity solution of $(P)$ solves the obstacle problem (2.1). This completes the proof of Theorem 3.1 for initial data satisfying (3.8).

Lemma 3.11. Let $v$ be a viscosity solution of $(P)$ with initial data $v_{0}$, and let $u$ be the unique solution of (2.1). If $v_{0}$ satisfies (3.8), then

$$
\int_{0}^{t} v(x, s) d t=\int_{0}^{t} v^{*}(x, s) d s=u(x, t) .
$$

In particular if $u$ is differentiable with respect to $t$, then $u_{t}=v=v^{*}$. 
Proof. Equation (3.10) yields, for all $t \geq h$ :

$$
\frac{u(x, t)-u(x, t-h)}{h} \leq(1+\delta) v(x, t), \quad \text { with } \delta(h) \rightarrow 0 \text { as } h \rightarrow 0 .
$$

Hence

$$
(1+\delta) \int_{h}^{t} v(x, s) d s \geq \frac{1}{h} \int_{t-h}^{t} u(x, s) d s-\frac{1}{h} \int_{0}^{h} u(x, s) d s .
$$

Sending $h$ to 0 , keeping in mind that $u(x, 0)=0$ and $u$ is continuous, we deduce

$$
\int_{0}^{t} v(x, s) d s \geq u(x, t)
$$

Observe that

$$
(1+\delta) \frac{u(x, t+h)-u(x, t)}{h} \geq v(x, t), \quad \text { with } \delta(h) \rightarrow 0 \text { as } h \rightarrow 0 .
$$

As a matter of fact, this will be a consequence of the maximum principle if we can show that it holds at $t=0$. So we have to check that $(1+\delta) u(x+h) \geq h v_{0}(x)$ for small $h$, but this follows from the fact that $t v_{0}(x) /(1+\delta)$ is a subsolution of (2.1) for small time, since

$$
\left(\partial_{t}-\mathcal{A}\right)\left(t v_{0}(x)\right)=v_{0}(x)-t \mathcal{A} v_{0}(x) \leq(1+\delta) v_{0}(x)
$$

for small times (using the fact that $\mathcal{A} v_{0} \geq 0$ near $\partial\left\{v_{0}>0\right\}$; see (3.8)).

Proceeding as before, it is now easy to check that (3.11) yields

$$
\int_{0}^{t} v^{*}(x, s) d s \leq u(x, t),
$$

and we conclude the proof after noticing that $v \leq v^{*}$.

Step 2 (General initial data). For general $v_{0}$ that do not satisfy (3.8), using Lemma 3.10, one can still construct a decreasing sequence $v_{0}^{k}$ satisfying (3.8) for some $\delta>0$ and $h>0$ and

$$
v_{0} \prec v_{0}^{k} \leq v_{0}+\frac{1}{k}, \quad \operatorname{supp} v_{0}^{k} \rightarrow \operatorname{supp} v_{0} \text { as } k \rightarrow \infty
$$

(where the convergence of the support holds with respect to the Hausdorff distance).

The proof in step 1 then applies, and the conclusions of Theorem 3.1 thus hold for the function $v_{k}(x, t)$, the solution of (3.1) with $u=u_{k}$, where $u_{k}$ solves (2.1) with $v_{0}^{k}$ instead of $v_{0}$.

Now let $v$ be the solution of (3.1) (with $u$ this time) and define

$$
\underline{v}(x, t):=\lim _{\varepsilon \rightarrow 0} \inf _{|(y, s)-(x, t)|, \frac{1}{k} \leq \varepsilon} v_{k}(y, s)
$$

and

$$
\bar{v}(x, t):=\lim _{\varepsilon \rightarrow 0} \sup _{|(y, s)-(x, t)|, \frac{1}{k} \leq \varepsilon} v_{k}(y, s) .
$$

Clearly $v_{k} \geq v$, and thus (recalling that $v$ is lower semicontinuous):

$$
\underline{v} \geq v \text { and } v^{*} \leq \bar{v} .
$$

Standard stability properties of viscosity solutions imply that $\bar{v}$ is a subsolution of $(P)$ and $\underline{v}$ is a supersolution of $(P)$. Furthermore, proceeding as in the proof of Lemma 3.8, we can show that

$$
\bar{v}(\cdot, 0)=v_{0} .
$$


A parallel argument, using a sequence of smaller initial data $w_{0}^{k} \prec v_{0}$ converging to $v_{0}$ and the corresponding solutions $\tilde{u}_{k}$ of the obstacle problem, generates another viscosity subsolution and supersolution $\bar{w}$ and $\underline{w}$ of $(P)$ with initial data $v_{0}$. Note that in this case, $\underline{w} \leq \bar{w} \leq v^{*}$.

Now we would like to show that $\underline{v}=\underline{w}$ using the uniqueness of the obstacle solution and Lemma 3.11 .

Lemma 3.11 implies that

$$
u_{k}=\int_{0}^{t} v_{k}(x, s) d s \quad \text { and } \quad \tilde{u}_{k}=\int_{0}^{t} w_{k}(x, s) d s,
$$

and Lemma 3.7 gives that both $u_{k}$ and $\tilde{u}_{k}$ converge locally uniformly to $u$ (where $u$ solves the obstacle problem (2.1) with initial condition $v_{0}(x)$ ).

The comparison principle for the viscosity solution of the Stefan problem (Theorem [2.8) implies that $\bar{v} \prec v_{k}$ and $w_{k} \prec \underline{w}$ for every $k$. Therefore

$$
\int_{0}^{t} \bar{v}(x, s) d s \leq \int_{0}^{t} v_{k}(x, s) d s=u_{k}(x, t)
$$

and

$$
\tilde{u}_{k}(x, t)=\int_{0}^{t} w_{k}(x, s) d s \leq \int_{0}^{t} \underline{w}(x, s) d s .
$$

Taking the limit $k \rightarrow \infty$ yields

$$
\int_{0}^{t} \bar{v}(x, s) d s \leq u(x, t) \leq \int_{0}^{t} \underline{w}(x, s) d s \leq \int_{0}^{t} \bar{w}(x, s) d s .
$$

Since $\underline{w} \leq v \leq \underline{v}$, it follows that $\underline{v}(\cdot, t)=\bar{v}(\cdot, t)=v(\cdot, t)=\underline{w}(\cdot, t)$ for almost every $t>0$. In particular

$$
\int_{0}^{t} v(x, s) d s=u(x, t) \text { for all } x \text { and } t .
$$

Let us finish by showing that $v$ is a viscosity solution of $(P)$. Recall that by definition $v$ is continuous in $\Omega(v)$. Therefore from the previous argument and due to the lower semi-continuity of $\underline{v}$, one sees that $\underline{v} \leq v$ in $\Omega(v)$ (and thus in $\mathcal{O} \times(0, T)$ ). Similarly, we obtain $v^{*} \leq \bar{w}$ in $\Omega(v)$, but $v^{*}$ may be positive on $\Gamma(v)$. However, by construction of $v$,

$$
v^{*}(x, t)=\limsup _{(y, s) \rightarrow(x, t),(y, s) \in \Omega(v)} v(y, s) .
$$

As a result, $v^{*} \leq \bar{w}$ on $\Gamma(v)$ and

$$
v^{*}=\bar{w} \quad \text { and } \quad v=\underline{v},
$$

and in particular $v$ is a viscosity solution of $(P)$.

To conclude this section, we note that (3.13) actually yields a general comparison principle and uniqueness result:

Corollary 3.12 (General comparison principle). Let $u$ and $w$ be, respectively, $a$ viscosity subsolution and supersolution of $(P)$ with continuous initial data $u_{0} \leq w_{0}$. In addition suppose that $w_{0}$ (or $u_{0}$ ) satisfies (1.2). Then

$$
u_{*} \leq w \quad \text { and } \quad u \leq w^{*} \text { in } \mathbb{R}^{n} \times[0, \infty) .
$$


Proof.

1. Suppose $w_{0}$ satisfies (1.2). Define $v$ by (3.1), which solves (2.1) with $v_{0}$ replaced by $w_{0}$. Also define $v_{k}$ and $w_{k}$ as in step 2 of the proof of Theorem 3.1 but using the initial data $w_{0}$. By Theorem 2.8, $u \prec v_{k}$ for any $k$ and thus

$$
u_{*} \leq \underline{v}=v,
$$

and

$$
u \leq \bar{v}
$$

Moreover, thanks to equality (3.12), $v(\cdot, t)=\bar{v}(\cdot, t)$ for almost every $t$, and thus

$$
u(\cdot, t) \leq v(\cdot, t) \quad \text { for almost all } t \in \mathbb{R}_{+} .
$$

2. We now want to show that (3.15) implies that

$$
u \leq v^{*} \text {. }
$$

Let $\left(x_{0}, t_{0}\right) \in \mathbb{R}^{n} \times(0, \infty)$ and let $t_{k}$ be an increasing sequence such that

$$
t_{k}<t_{0}, \quad \lim t_{k}=t_{0}, \quad u\left(\cdot, t_{k}\right) \leq v\left(\cdot, t_{k}\right) \quad \text { for all } k
$$

(such a sequence exists in view of (3.15)). By definition of $v^{*}$, for all $\delta>0$, there exists $r>0$ such that if $\left|t_{k}-t_{0}\right| \leq r$, then

$$
v\left(x, t_{k}\right) \leq v^{*}\left(x_{0}, t_{0}\right)+\delta \quad \text { for all } x \in B_{r}\left(x_{0}\right)
$$

and therefore

$$
\alpha_{k}:=\sup _{x \in B_{r}\left(x_{0}\right)} u\left(x, t_{k}\right) \leq v^{*}\left(x_{0}, t_{0}\right)+\delta
$$

for all $k$ such that $\left|t_{k}-t_{0}\right| \leq r$. Finally, define

$$
h(x, t)=\alpha_{k}+\delta+\frac{M}{r^{2}}\left(\beta\left(x-x_{0}\right)^{2}+t-t_{k}\right) .
$$

It is easy to see that for $\beta$ small enough, $\left(\partial_{t}-\mathcal{A}\right) h \geq 0$ and

$$
\begin{array}{ll}
h\left(x, t_{k}\right)>\alpha_{k} \geq u\left(x, t_{k}\right) & \text { for } x \in B_{r}\left(x_{0}\right), \\
h(x, t) \geq \alpha_{k}+M \beta & \text { for } x \in \partial B_{r}\left(x_{0}\right), t \in\left(t_{k}, t_{0}\right),
\end{array}
$$

and so $h(x, t) \geq u(x, t)$ on the parabolic boundary of $B_{r}\left(x_{0}\right) \times\left(t_{k}, t_{0}\right)$ if $M$ is large enough (we can take $M \beta=\|u\|_{L^{\infty}\left(\mathbb{R}^{n} \times(0, \infty)\right)}$ ). By definition of subsolutions (using the fact that $h>0$ ),

$$
u\left(x_{0}, t_{0}\right) \leq h\left(x_{0}, t_{0}\right)=\alpha_{k}+\delta+\frac{M}{r^{2}}\left(t_{0}-t_{k}\right),
$$

and so (3.17) implies that

$$
u\left(x_{0}, t_{0}\right) \leq v^{*}\left(x_{0}, t_{0}\right)+2 \delta+\frac{M}{r^{2}}\left(t_{0}-t_{k}\right) .
$$

Since this inequality holds as soon as $\left|t_{k}-t_{0}\right| \leq r$ with $M$ independent of $t_{k}$, we deduce that

$$
u\left(x_{0}, t_{0}\right) \leq v^{*}\left(x_{0}, t_{0}\right)+\delta
$$

and letting $\delta$ go to zero, (3.16) follows. 
3. Similarly, we have $w_{k} \prec w$ for all $k$ and thus, due to (3.13),

$$
w=w_{*} \geq \underline{w} \quad \text { and } w^{*} \geq \bar{w}=v^{*} .
$$

Moreover, due to the equality (3.12), $v(\cdot, t)=\underline{w}(\cdot, t)$ for almost every $t$, and proceeding as above it follows that

$$
w \geq v \quad \text { and } \quad w^{*} \geq v^{*}
$$

Corollary 3.12 is now a consequence of (3.14), (3.16) and (3.18).

As a consequence of this comparison principle the following uniqueness result holds:

Corollary 3.13. Let $v_{0}$ satisfy (1.2). Then there exists a unique viscosity solution $v$ of $(P)$. Moreover $v$ is given by the formula (3.1).

Remark 3.14. When $a_{i j}=\delta_{i j}$ (i.e. for the classical Stefan problem), Caffarelli and Friedman $[\mathrm{CF}]$ show that $u_{t}$ is continuous in space and time. In particular $u_{t}=0$ on $\Gamma(u)$. In this case the proof of Theorem 3.1 could be simplified and we would have $v=u_{t}$. By a change of coordinates, the continuity of $v$ could also be established when the coefficients $\left(a_{i j}\right)$ are constant. In particular the homogenized solution $u^{0}(x, t)$ in the next section has continuous time derivative $v^{0}(x, t)$.

\section{Homogenization of the VARiational PROBlem}

The last two sections of this paper are devoted to the homogenization of the Stefan problem $\left(P^{\varepsilon}\right)$. In this section the investigation is on the homogenization of the variational problem corresponding to $\left(P^{\varepsilon}\right)$. The main focus of the analysis will be on the random case (hypothesis (b2)), since the periodic case is a little bit easier and was already studied by Rodrigues in [R2].

More precisely, we assume that the coefficients $a_{i j}(y, \omega)$ satisfy hypotheses (a) and (b2) stated in the introduction, and we consider $u^{\varepsilon}(x, t, \omega)$, a solution of the parabolic variational inequality associated to the Stefan problem with oscillating coefficients: For a.e. $\omega \in \Omega$, let $u^{\varepsilon} \in L^{2}\left(0, T ; H^{1}(\mathcal{O})\right)$ with $\partial_{t} u^{\varepsilon} \in L^{2}(\mathcal{O} \times[0, T])$ be the unique solution of

$$
\left\{\begin{array}{l}
u^{\varepsilon}(\cdot, t) \in \mathcal{K}(t), \\
\int_{\mathcal{O}} \partial_{t} u^{\varepsilon}\left(v-u^{\varepsilon}\right) d x+a^{\varepsilon}\left(u^{\varepsilon}, v-u^{\varepsilon}\right) \geq \int f\left(v-u^{\varepsilon}\right) d x, \quad \forall v \in \mathcal{K}(t)
\end{array}\right.
$$

for a.a. $t \in(0, T)$, with $u^{\varepsilon}(x, 0)=0$.

Recall that

$$
\begin{gathered}
\mathcal{K}(t)=\left\{v \in H_{0}^{1}(\mathcal{O}) ; v \geq 0\right\}, \\
f(x)= \begin{cases}v_{0}(x) & \text { for } x \in \Omega_{0}, \\
-1 & \text { for } x \notin \Omega_{0} .\end{cases}
\end{gathered}
$$

Finally we introduce the bilinear form

$$
a^{\varepsilon}(u, v)=\int_{\mathcal{O}} a_{i j}(x / \varepsilon) D_{i} u D_{j} v d x .
$$

The goal in this section is to prove the uniform convergence of $u^{\varepsilon}(x, t, \omega)$, the solution of the variational inequality (4.1), to the solution $u^{0}(x, t)$ of some homogenized variational problem. 
The homogenization of variational inequalities, of elliptic or parabolic type, is a classical problem which has been addressed in numerous papers, in particular in the periodic case. The main references for the homogenization of elliptic variational inequalities in the case of random coefficients are the papers of G. Dal Maso and L. Modica DM1-DM2. Their results rely on the notion of $\Gamma$-convergence and make use of the subadditive ergodic theorem of M. A. Akcoglu and U. Krengel AK to show the existence of a homogenized functional independent of $\omega$. Since the authors could not find a reference that addresses the case of the parabolic inequality in the random case, for the sake of completeness, a detailed proof will be given for the results we need, using [DM1]-[DM2] and the notion of $\Gamma$-convergence (see the monograph of G. Dal Maso Da for an introduction to $\Gamma$-convergence).

Theorem 4.1. Let $\left(a_{i j}(x, \omega)\right)_{i j}$ be a given symmetric matrix satisfying (1.1) and assume that the coefficients $a_{i j}(x, \omega)$ satisfy hypotheses (a) and (b2).

For $\varepsilon>0$, let $u^{\varepsilon}$ be the unique solution of (4.1). Then $u^{\varepsilon}(x, t, \omega)$ converges uniformly with respect to $(x, t)$ and for all $\omega \in \tilde{\Omega}$ to $u^{0}(x, t)$, the solution of

$$
\left\{\begin{array}{l}
u^{0}(t) \in \mathcal{K}(t), \\
\int_{\mathcal{O}} \partial_{t} u^{0}\left(v-u^{0}\right) d x+a^{0}\left(u^{0}, v-u^{0}\right) \geq \int f\left(v-u^{0}\right) d x \quad \forall v \in \mathcal{K}(t)
\end{array}\right.
$$

with $u^{0}(x, 0)=0$, and where $a^{0}(u, v)$ is a bilinear form defined later on.

Naturally, a similar result holds in the periodic case (hypothesis (b1)) (see [R2] for details). Note that (4.2) is exactly the obstacle problem associated to the homogenized Stefan problem $\left(P^{0}\right)$.

We define the following functional:

$$
\mathscr{J}_{\varepsilon}(v):=a^{\varepsilon}(v, v)=\int_{\mathcal{O}} a_{i j}(x / \varepsilon, \omega) D_{i} v D_{j} v d x \quad \text { for } v \in H^{1}(\mathcal{O}) .
$$

The homogenization of functionals such as $\mathscr{J}_{\varepsilon}$ under the hypothesis of stationary ergodicity has been studied, in particular, by G. Dal Maso and L. Modica DM1, DM2. It relies on the notion of $\Gamma$-convergence introduced by De Giorgi. We take the following definition of $\Gamma(X)$-convergence (Dal Maso [Da]):

Definition 4.2. Let $X$ be a topological space. A sequence of functionals $F_{h}$ is said to $\Gamma(X)$-converge to $F$ if the following conditions are satisfied:

(i) For every $u \in X$ and for every $\left(u_{h}\right)$ converging to $u$ in $X$, we have

$$
F(u) \leq \liminf _{h \rightarrow 0} F_{h}\left(u_{h}\right) .
$$

(i) For every $u \in X$ there exists a sequence $\left(u_{h}\right)$ converging to $u$ in $X$, such that

$$
F(u)=\lim _{h \rightarrow 0} F_{h}\left(u_{h}\right) .
$$

The key result is the following theorem:

Theorem 4.3 (G. Dal Maso, L. Modica DM1-DM2]). The functionals $\mathscr{J}_{\varepsilon} \Gamma\left(L^{2}\right)$ converge $P$-almost everywhere as $\varepsilon \rightarrow 0$ to a functional $\mathscr{J}_{0}$, where $\mathscr{J}_{0}$ is a quadratic functional independent of $\omega$ of the form

$$
\mathscr{J}_{0}(u)=\int q_{i j} D_{i} u D_{j} u
$$


where the coefficients $q_{i j}$ are constant and satisfy

$$
\lambda^{\prime}|\xi|^{2} \leq \sum_{i j} q_{i j} \xi_{i} \xi_{j} \leq \Lambda^{\prime}|\xi|^{2} .
$$

In the periodic case, it is well known that the homogenized coefficients $q_{i j}$ are defined by (see $[\mathrm{BLP}]$ ):

$$
q_{i j}=\int_{Y} a_{k l}(y) D_{k}\left[\chi^{i}(y)-y_{i}\right] D_{l}\left[\chi^{j}(y)-y_{j}\right] d y=a\left(\chi^{i}-y_{i}, \chi^{j}-y_{j}\right),
$$

where the $\chi^{i}$ are $Y$-periodic functions defined via the following cell problem:

$$
a\left(\chi^{i}-y_{i}, \psi\right)=0 \text { for all } Y \text {-periodic } \psi \text {. }
$$

In the random case, we refer to G. Papanicolaou and S.R.S. Varadhan [PV] for a corresponding formula.

Note that $\mathscr{J}_{\varepsilon}$ is only well defined for $u \in H^{1}$. In the proposition above, we thus implicitly defined (following Dal Maso $[\mathrm{Da}$ )

$$
\mathscr{J}_{\varepsilon}(u)=\infty \quad \text { for } u \in L^{2} \backslash H^{1} .
$$

Furthermore, if we denote

$$
\mathscr{J}_{\varepsilon}^{0}(u)= \begin{cases}\mathscr{J}_{\varepsilon}(u), & \text { if } u \in H_{0}^{1}(\mathcal{O}), \\ +\infty, & \text { otherwise }\end{cases}
$$

then ([Da], Theorem 21.1):

Corollary 4.4. The sequence of functionals $\mathscr{J}_{\varepsilon}^{0} \Gamma$-converges to $\mathscr{J}_{0}^{0}$ in $L^{2}(\mathcal{O})$.

From now on, $\widetilde{\Omega} \subset \Omega$ will denote the subset of the probability set such that $P(\widetilde{\Omega})=1$ and the $\mathscr{J}_{\varepsilon} \Gamma$-converge to $\mathscr{J}_{0}$ for all $\omega \in \widetilde{\Omega}$.

In order to prove this result, a couple of intermediate lemmas are necessary:

Lemma 4.5. Let $t>0$. For all $w \in \mathcal{K}(t)$ there exists a sequence $w^{\varepsilon}$ of functions in $\mathcal{K}(t)$ that converges to $w$ in $L^{2}(\mathcal{O})$-strong (and $H^{1}(\mathcal{O})$-weak), and such that

$$
\mathscr{J}_{0}(w)=\lim _{\varepsilon \rightarrow 0} \mathscr{J}_{\varepsilon}\left(w^{\varepsilon}\right) .
$$

Proof. If $w \in \mathcal{K}(t)$, then we have in particular that $w \in H_{0}^{1}$. Therefore Corollary 4.4 implies that there exists a sequence $w^{\varepsilon}$ that converges to $w$ in $L^{2}(\mathcal{O})$-strong and such that

$$
\mathscr{J}_{0}(w)=\mathscr{J}_{0}^{0}(w)=\lim _{\varepsilon \rightarrow 0} \mathscr{J}_{\varepsilon}^{0}\left(w^{\varepsilon}\right) .
$$

Since $w \in H_{0}^{1}(\mathcal{O}), \mathscr{J}_{0}^{0}(w)<\infty$ and so $\mathscr{J}_{\varepsilon}^{0}\left(w^{\varepsilon}\right)<\infty$ for $\varepsilon$ small enough. This implies in particular that $w^{\varepsilon} \in H_{0}^{1}(\mathcal{O})$, so $w^{\varepsilon}=0$ on $\partial \mathcal{O}$ and $\mathscr{J}_{\varepsilon}^{0}\left(w^{\varepsilon}\right)=\mathscr{J}_{\varepsilon}\left(w^{\varepsilon}\right)$. (Note that the ellipticity of $a_{i j}$ implies that $w^{\varepsilon}$ converges $H^{1}$-weak.)

Next, one needs to check if it is possible to choose $w^{\varepsilon} \geq 0$. For that purpose, let us set

$$
\tilde{w}^{\varepsilon}:=w_{+}^{\varepsilon}+w_{-}^{\varepsilon} \geq 0 .
$$

Then $\nabla \tilde{w}^{\varepsilon}=\nabla w^{\varepsilon} \chi_{w^{\varepsilon}>0}-\nabla w^{\varepsilon} \chi_{w^{\varepsilon}<0}$ and therefore

$$
\mathscr{J}_{\varepsilon}\left(\tilde{w}^{\varepsilon}\right)=\mathscr{J}_{\varepsilon}\left(w^{\varepsilon}\right) \longrightarrow \mathscr{J}_{0}(w) \quad \text { as } \varepsilon \rightarrow 0 .
$$

Finally, we have

$$
\tilde{w}^{\varepsilon}-w^{\varepsilon}=2 w_{-}^{\varepsilon}=\left|w^{\varepsilon}\right|-w^{\varepsilon} \longrightarrow|w|-w, \quad \text { in } L^{2}(\mathcal{O}) \text {-strong. }
$$


But $|w|-w=0$ a.e. since $w \geq 0$, and so $\tilde{w}^{\varepsilon}$ converges to $w$ strongly in $L^{2}$ (and weakly in $H^{1}$ ).

Now we are ready to prove Theorem 4.1 ,

Proof of Theorem 4.1. Throughout the proof, $\omega \in \widetilde{\Omega}$ will remain fixed.

1. Classical parabolic estimates give that $u^{\varepsilon}$ is bounded in $L^{2}\left(0, T ; H^{1}\right) \cap$ $L^{\infty}\left(0, T, L^{2}\right)$ and $\partial_{t} u^{\varepsilon}$ is bounded in $L^{2}\left(0, T, L^{2}\right)$. In particular, $u^{\varepsilon}$ is in a compact set of $L^{\infty}\left(0, T, L^{2}\right)$. Furthermore, minor adaptations from [FK] (see also [R2]) give

$$
0 \leq \partial_{t} u^{\varepsilon} \leq C \quad \text { a.e. } \mathcal{O} \times(0, T) .
$$

Consider a subsequence $u^{\varepsilon}$ that converges to $\bar{u}$ in $L^{\infty}\left(0, T, L^{2}\right)$. We can always assume that $\partial_{t} u^{\varepsilon}$ converges to $\partial_{t} \bar{u}$ in $L^{\infty}((0, T) \times \mathcal{O})$-weak ${ }^{*}$, and proceeding as in [R2, one can verify that $u^{\varepsilon}$ is bounded in $\mathcal{C}^{\alpha, \alpha / 2}$ and therefore that the convergence of $u^{\varepsilon}$ to $\bar{u}$ is uniform in $x$ and $t$.

2. Next, note that (4.1) is equivalent to

$$
\begin{aligned}
& \left(\partial_{t} u^{\varepsilon}, v-u^{\varepsilon}\right)+\frac{1}{2} \mathscr{J}_{\varepsilon}(v) \geq \frac{1}{2} \mathscr{J}_{\varepsilon}\left(u^{\varepsilon}\right)+\left(f, v-u^{\varepsilon}\right) \quad \forall v \in \mathcal{K}(t), \\
& u^{\varepsilon}(t) \in \mathcal{K}(t)
\end{aligned}
$$

(and a similar equivalence holds for (4.2)). As a matter of fact, it is readily seen that (4.1) and the fact that $a^{\varepsilon}(u, v-u) \leq \frac{1}{2} \mathscr{J}_{\varepsilon}(v)-\frac{1}{2} \mathscr{J}_{\varepsilon}(u)$ for any functions $u$, $v$ implies (4.3). On the other hand, taking $v=u^{\varepsilon}+\delta\left(w-u^{\varepsilon}\right)$ in (4.3) ( $v$ belongs to $\mathcal{K}$ for any $w \in \mathcal{K}$ if $\delta<1$ ) and passing to the limit $\delta \rightarrow 0$ implies (4.1).

3. Now let $v \in \mathcal{K}(t)$. Due to Lemma 4.5, there exists a sequence of functions $v^{\varepsilon}$ in $\mathcal{K}(t)$ that converges to $v$ in $L^{2}(\mathcal{O})$-strong and such that

$$
\lim _{\varepsilon \rightarrow 0} \mathscr{J}_{\varepsilon}\left(v^{\varepsilon}\right)=\mathscr{J}_{0}(v)
$$

Using (4.3) with $v=v^{\varepsilon}$ and passing to the limit $\varepsilon \rightarrow 0$, we deduce that

$$
\begin{aligned}
& \left(\partial_{t} \bar{u}, v-\bar{u}\right)+\frac{1}{2} \mathscr{J}_{0}(v) \geq \frac{1}{2} \mathscr{J}_{0}(\bar{u})+(f, v-\bar{u}) \quad \forall v \in \mathcal{K}(t), \\
& \bar{u}(t) \in \mathcal{K}(t),
\end{aligned}
$$

for a.a. $t \in(0, T)$, which is equivalent to (4.2). The uniqueness of $u^{0}$ implies that $\bar{u}=u^{0}$ and gives that the whole sequence $u^{\varepsilon}$ converges to $u^{0}$.

Finally, recall (see [Da] ) that the $\Gamma$-convergence of the functional $\mathscr{J}_{\varepsilon}$ implies the $G$-convergence of the corresponding elliptic operator. More precisely, let $\mathcal{A}^{\varepsilon}$ and $\mathcal{A}^{0}$ denote the elliptic operators respectively corresponding to the functionals $\mathscr{J}_{\varepsilon}$ and $\mathscr{J}_{0}$ :

$$
\mathcal{A}^{\varepsilon}(u)=D_{i}\left(a_{i j}(x / \varepsilon, \omega) D_{j} u\right), \quad \mathcal{A}^{0}(u)=D_{i}\left(q_{i j} D_{j} u\right) .
$$

Proposition 4.6. For any $\alpha \geq 0$, let $u^{\varepsilon} \in H^{1}$ be the solution of

$$
\mathcal{A}^{\varepsilon} u^{\varepsilon}+\alpha u^{\varepsilon}=f
$$

in $B_{r}$ with $u^{\varepsilon}=g$ on $\partial B_{r}$, and let $u \in H^{1}$ be the solution of

$$
\mathcal{A}^{0} u+\alpha u=f
$$

in $B_{r}$ with $u=g$ on $\partial B_{r}$. Then $u^{\varepsilon}(x, \omega)$ converges to $u(x)$ strongly in $L^{2}$, and uniformly in $x$, for all $\omega \in \tilde{\Omega}$. 
The following proposition now follows from Proposition 4.6 and Trotter-Kato's formula:

Proposition 4.7. Let $u^{\varepsilon} \in L^{2}\left(0, \tau, H^{1}\left(B_{r}\right)\right)$ be a solution of

$$
\partial_{t} u^{\varepsilon}+\mathcal{A}^{\varepsilon} u^{\varepsilon}=f
$$

in $B_{r} \times(0, \tau)$ with boundary condition $u^{\varepsilon}=g$ on the parabolic boundary $\partial B_{r} \times$ $(0, \tau) \cup B_{r} \times\{0\}$. Then, $u^{\varepsilon}(x, t, \omega)$ converges uniformly in $x$ and $t$ to $u^{0}(x, t)$, the solution of

$$
\partial_{t} u^{0}+\mathcal{A}^{0} u^{0}=f
$$

in $B_{r} \times(0, \tau)$.

\section{Homogenization of the Stefan problem}

In this last section, the proof of the homogenization result is completed by showing the uniform convergence of the solution of $\left(P^{\varepsilon}\right)$ to the solution of $\left(P^{0}\right)$.

Let $u^{\varepsilon}(x, t)$ solve (4.1) and let $v^{\varepsilon}(x, t)$ be the corresponding solution of $\left(P^{\varepsilon}\right)$ given by Theorem 3.1. Now define $u^{0}(x, t)$ as the limit of $u^{\varepsilon}(x, t)$ given by Theorem 4.1 , and let $v^{0}(x, t)$ solve

$$
\left\{\begin{array}{l}
\partial_{t} v^{0}-\mathcal{A}^{0} v^{0}=0 \quad \text { in } \Omega_{t}\left(u^{0}\right), \\
v^{0}(x, 0)=v_{0}(x) .
\end{array}\right.
$$

Thanks to Theorem 3.1 the function $v^{0}(x, t)$ then solves the Stefan free boundary problem $\left(P^{0}\right)$ with initial data $v_{0}(x)$. Recall that $\mathcal{A}^{0}$ is the elliptic operator corresponding to the functional $\mathscr{J}_{0}$ defined by Theorem 4.3

$$
\mathcal{A}^{0} u=D_{i}\left(q_{i j} D_{j} u\right) .
$$

In particular, Remark 3.14 yields

Lemma 5.1. The function $v^{0}(x, t)=\left(\partial_{t} u^{0}\right)(x, t)$ is continuous with respect to $(x, t)$.

The goal of this section is to prove:

Theorem 5.2. Suppose $v_{0}$ satisfies (1.2). Then the solution $v^{\varepsilon}(x, t)$ of $\left(P^{\varepsilon}\right)$ locally uniformly converges to the solution $v^{0}(x, t)$ of $\left(P^{0}\right)$. Moreover $\Gamma\left(v^{\varepsilon}\right)$ locally uniformly converges to $\Gamma\left(v^{0}\right)$ with respect to the Hausdorff distance.

In order to prove the main theorem, let us define

$$
v^{*}(x, t):=\limsup v^{\varepsilon}(x, t):=\limsup _{(y, s), \varepsilon \rightarrow(x, t), 0} v^{\varepsilon}(y, s)
$$

and

$$
v_{*}(x, t):=\liminf _{*} v^{\varepsilon}(x, t):=\liminf _{(y, s), \varepsilon \rightarrow(x, t), 0} v^{\varepsilon}(y, x) .
$$

To obtain the uniform convergence of $v^{\varepsilon}$, it suffices to show that

$$
v_{*}=v^{*}=v^{0} .
$$

The following proposition summarizes the properties of $\mathcal{A}^{0}$ that we will need. 


\section{Proposition 5.3.}

(a) The operator $\mathcal{A}^{0}$ is uniformly elliptic with constant coefficient.

(b) If $w^{\varepsilon}$ satisfies

$$
w_{t}^{\varepsilon}-\mathcal{A}^{\varepsilon} w^{\varepsilon}=0 \quad \text { in } \Sigma,
$$

then the functions

$$
w^{*}=\lim { }^{*} \sup w^{\varepsilon} \quad \text { and } \quad w_{*}=\liminf _{*} w^{\varepsilon}
$$

are respectively the subsolution and supersolution of

$$
w_{t}-\mathcal{A}^{0} w=0 \quad \text { in } \Sigma .
$$

Proof. The first part follows from Theorem 4.3. The second part will be a consequence of Proposition 4.7. As a matter of fact, if $w^{*}$ is not a subsolution of (5.2), then there exists a function $\varphi(x, t)$ which touches $w^{*}$ from above in a parabolic neighborhood $Q_{r}\left(x_{0}, t_{0}\right):=B_{r}\left(x_{0}\right) \times\left(t_{0}-r^{2}, t_{0}\right)$ of a point $\left(x_{0}, t_{0}\right) \in \Sigma$ and satisfies

$$
\varphi_{t}-\mathcal{A}^{0} \varphi>0 \text { in } Q_{r}\left(x_{0}, t_{0}\right) .
$$

By considering a smooth perturbation of $\varphi$ instead of $\varphi$, we may assume

$$
\varphi(x, t)-w^{*}(x, t)>\delta \quad \text { for }(x, t) \in \partial_{p} Q_{r}\left(x_{0}, t_{0}\right)
$$

and

$$
\varphi(x, t) \leq w^{*}\left(x_{0}, t_{0}\right)-\delta \quad \text { for }(x, t) \in B_{\delta}\left(x_{0}, t_{0}\right) \times\left(t_{0}-\delta, t_{0}\right)
$$

for some small $\delta>0$.

We now define $\varphi^{\varepsilon}$ to be the solution of

$$
\varphi_{t}^{\varepsilon}-\mathcal{A}^{\varepsilon} \varphi^{\varepsilon}=\varphi_{t}-\mathcal{A}^{0} \varphi \geq 0 \quad \text { in } Q_{r}\left(x_{0}, t_{0}\right)
$$

with $\varphi^{\varepsilon}=\varphi$ on the parabolic boundary of $Q_{r}\left(x_{0}, t_{0}\right)$. Proposition 4.7 then yields that $\varphi^{\varepsilon}$ uniformly converges to $\varphi$ as $\varepsilon \rightarrow 0$.

Finally, (5.3) implies that

$$
\varphi^{\varepsilon}(x, t)>w^{\varepsilon}(x, t) \quad \text { on } \partial_{p} Q_{r}\left(x_{0}, t_{0}\right)
$$

for $\varepsilon$ small enough (we recall that $w^{*}$ is the limsup) and (5.4) yields that for a small $\varepsilon>0$ there exists $\left(x_{1}, t_{1}\right) \in Q_{r}\left(x_{0}, t_{0}\right)$ such that

$$
\varphi^{\varepsilon}\left(x_{1}, t_{1}\right)<w^{\varepsilon}\left(x_{1}, t_{1}\right) .
$$

Since $w^{\varepsilon}$ and $\varphi^{\varepsilon}$ are respectively the solution and supersolution of (5.1), we get a contradiction.

In order to prove the main theorem, we need several lemmas which describe the relationship between $v^{\varepsilon}, v^{*}$ and $v^{0}$.

Lemma 5.4. Suppose $\left(x_{k}, t_{k}\right) \in\left\{u^{\varepsilon_{k}}=0\right\}$ and $\left(x_{k}, t_{k}, \varepsilon_{k}\right) \rightarrow\left(x_{0}, t_{0}, 0\right)$. Then $u^{0}\left(x_{0}, t_{0}\right)=0$. In particular if $x_{k} \in \Gamma_{t_{k}}\left(u^{\varepsilon_{k}}\right)$, then $x_{0} \in \Gamma_{t_{0}}\left(u^{0}\right)$.

Proof. The uniform convergence and the continuity of $u^{\varepsilon}$ easily give the first part.

If moreover $x_{k} \in \Gamma_{t_{k}}\left(u^{\varepsilon_{k}}\right)$ for all $k$, then the nondegeneracy estimate (Lemma 3.3) gives, for any small $r>0$, the existence of $y_{k} \in B_{r}\left(x_{k}\right)$ such that

$$
u^{\varepsilon_{k}}\left(y_{k}, t_{k}\right) \geq c r^{2} \text {. }
$$


Up to a subsequence, we can now assume that $y_{k} \longrightarrow y_{0} \in B_{r}\left(x_{0}\right)$ and the uniform convergence and continuity of $u^{\varepsilon}$ yields

$$
u^{0}\left(y_{0}, t_{0}\right) \geq c r^{2} \text {. }
$$

It follows that $B_{r}\left(x_{0}\right) \cap \Omega_{t_{0}}\left(u^{0}\right) \neq \emptyset$ for all $r>0$; hence $x_{0} \in \Gamma_{t_{0}}\left(u^{0}\right)$.

Lemma 5.5. The function $v_{*}(x, t)$ satisfies

$$
\Omega\left(v^{0}\right) \subset \Omega\left(v_{*}\right) .
$$

In particular $v_{*} \geq v^{0}$.

Proof.

1. Suppose $\left(x_{0}, t_{0}\right) \in \Omega\left(v^{0}\right)$. Since $v^{0}(x, t)$ is continuous, it follows that $\Sigma:=$ $B_{r}\left(x_{0}\right) \times\left[t_{0}-r, t_{0}\right] \subset \Omega\left(v^{0}\right)=\Omega\left(u^{0}\right)$ for some $r>0$ and so $u^{0}>0$ in $\Sigma$. Since $u^{\varepsilon}$ locally uniformly converges to $u^{0}$, it follows that $\Sigma \subset \Omega\left(u^{\varepsilon}\right)=\Omega\left(v^{\varepsilon}\right)$ for small $\varepsilon>0$.

2. Recall that we have $\int_{0}^{t} v^{\varepsilon}(x, s) d s=u^{\varepsilon}(x, t)$, and thus

$$
\int_{t_{0}-r}^{t_{0}} v^{\varepsilon}(x, s) d s=u^{\varepsilon}\left(x, t_{0}\right)-u^{\varepsilon}\left(x, t_{0}-r\right) .
$$

Suppose now that $v^{*}\left(x_{0}, t_{0}\right)=0$. Since $\Sigma$ lies in $\Omega\left(v^{\varepsilon}\right)$, $v^{\varepsilon}$ is a nonnegative solution of the uniformly parabolic equation $v_{t}^{\varepsilon}-A^{\varepsilon} v^{\varepsilon}=0$ in $\Sigma$. Therefore, the parabolic Harnack inequality (see Doob [Do and Moser [M] implies that $v^{\varepsilon}(x, s) \rightarrow$ 0 uniformly in $\Sigma$ as $\varepsilon \rightarrow 0$. Equality (5.5) therefore yields $u\left(x, t_{0}\right)=u\left(x, t_{0}-r\right)$ in $B_{r}\left(x_{0}\right)$, which contradicts Proposition 3.2 . Thus $\left(x_{0}, t_{0}\right)$ must be in $\Omega\left(v^{*}\right)$.

\section{Lemma 5.6.}

(i) For any $\left(x_{0}, t_{0}\right) \in \Gamma\left(v^{0}\right)$, there exists a sequence $\varepsilon_{k} \rightarrow 0$ and $\left(x_{k}, t_{k}\right) \in$ $\Gamma\left(v^{\varepsilon_{k}}\right)$ such that $\left(x_{k}, t_{k}, \varepsilon_{k}\right) \rightarrow\left(x_{0}, t_{0}, 0\right)$.

(ii) $\Gamma\left(v^{*}\right)$ is a subset of $\Gamma\left(v^{0}\right)$.

Proof.

1. We first prove (i). Suppose the result does not hold. Then there exists $\left(x_{0}, t_{0}\right) \in$ $\Gamma\left(v^{0}\right)$ and $r>0$ such that, for all $\varepsilon>0$,

$$
\Sigma:=B_{r}\left(x_{0}\right) \times\left[t_{0}-r, t_{0}+r\right] \subset \Omega\left(v^{\varepsilon}\right)
$$

or

$$
\Sigma \subset\left\{v^{\varepsilon}=0\right\} .
$$

In the latter case, $\Sigma \subset\left\{u^{\varepsilon}=0\right\}$ for all $\varepsilon>0$ and so $\Sigma \subset\left\{u^{0}=0\right\}=\left\{v^{0}=0\right\}$, which is impossible. Thus one may assume that (5.6) holds.

By Lemma 3.4 there exists $m>0$ such that

$$
B_{r / 4}\left(x_{0}\right) \cap \Omega_{t_{0}-2 m r^{2}}\left(v^{0}\right) \neq \emptyset,
$$

so there exists $y_{0} \in B_{r / 4}\left(x_{0}\right)$ such that

$$
v^{0}\left(y_{0}, t_{0}-2 m r^{2}\right)=c_{0}>0
$$

Since $v^{0} \leq v^{*}$ (by Lemma [5.5), it follows that there exists a sequence $\left(y_{k}, s_{k}, \varepsilon_{k}\right)$ which converges to $\left(y_{0}, t_{0}-2 m r^{2}, 0\right)$ and such that

$$
v^{\varepsilon_{k}}\left(y_{k}, s_{k}\right)>c_{0} / 2 \text {. }
$$


Due to (5.6) and the parabolic Harnack inequality, we thus get

$$
v^{\varepsilon_{k}}>c_{1} \text { in } \tilde{\Sigma}:=B_{r / 2}\left(y_{0}\right) \times\left[t_{0}-m r^{2}, t_{0}\right]
$$

for sufficiently large $k$ and where $c_{1}$ is independent of $\varepsilon>0$.

Using the fact that $\int_{0}^{t} v^{\varepsilon}(\cdot, s) d s=u^{\varepsilon}$, we deduce that

$$
u^{\varepsilon}>c_{1} m r^{2} \text { in } B_{r / 2}\left(y_{0}\right) \times\left\{t_{0}\right\}
$$

and the uniform convergence of $u^{\varepsilon}$ yields

$$
B_{r / 4}\left(x_{0}\right) \subset B_{r / 2}\left(y_{0}\right) \subset \Omega_{t_{0}}\left(u^{0}\right),
$$

thus contradicting the hypothesis.

2. Now to prove (ii), suppose $\left(x_{0}, t_{0}\right) \in \Gamma\left(v^{*}\right)$. Since $v^{0} \leq v^{*}$ by Lemma 5.5, $\left(x_{0}, t_{0}\right)$ lies in $\overline{\left\{v^{0}=0\right\}}=\left\{v^{0}=0\right\}$ (recall that $v^{0}$ is continuous).

Suppose now that $\left(x_{0}, t_{0}\right)$ lies in the interior of $\left\{v^{0}=0\right\}=\left\{u^{0}=0\right\}$. If there exists $\left(x_{\varepsilon}, t_{\varepsilon}\right) \in \bar{\Omega}\left(u^{\varepsilon}\right)$ which converges to $\left(x_{0}, t_{0}\right)$, then Lemma 3.3 applied to $u^{\varepsilon}$ yields a contradiction. Therefore there exists $r>0$ such that

$$
B_{r}\left(x_{0}\right) \times\left[t_{0}-r, t_{0}+r\right] \subset\left\{u^{\varepsilon}=0\right\}=\left\{v^{\varepsilon}=0\right\} \text { for sufficiently small } \varepsilon>0 .
$$

It follows that $v^{*}=0$ in $B_{r}\left(x_{0}\right) \times\left[t_{0}-r, t_{0}+r\right]$, yielding a contradiction. Therefore $\left(x_{0}, t_{0}\right)$ must belong to $\Gamma\left(v^{0}\right)$ and the lemma follows.

Proposition 5.7. The function $v^{*}$ is a subsolution of the limiting problem $\left(P_{0}\right)$.

Proof. Recall that $\left(v^{0}\right)^{*}=v^{0}$ is a subsolution of the limiting problem.

1. Since $v^{\varepsilon}$ is a subsolution of (5.2) in $\Omega\left(v^{\varepsilon}\right)$, arguments as in the proof of Proposition 5.3 lead us to conclude that $v^{*}$ is a subsolution of (5.2) in $\Omega\left(v^{*}\right)$.

It remains to check for the behavior of $v^{*}$ on its free boundary $\Gamma\left(v^{*}\right)$. Let $\phi$ be a smooth function in $C^{2,1}$ such that

$$
\begin{gathered}
v^{*} \leq \phi_{+}=\max (\phi, 0) \quad \text { in } \Sigma:=B_{r}\left(x_{0}\right) \times\left(t_{0}-\tau, t_{0}\right], \\
v^{*}\left(x_{0}, t_{0}\right)=\phi\left(x_{0}, t_{0}\right) \quad \text { with } \quad\left(x_{0}, t_{0}\right) \in \Gamma\left(v^{*}\right) .
\end{gathered}
$$

The claim is that

$$
\min \left(\left(\partial_{t} \phi-\mathcal{A}^{0} \phi\right)\left(x_{0}, t_{0}\right),\left(\phi_{t}-F^{0}(D \phi)|D \phi|\right)\left(x_{0}, t_{0}\right)\right) \leq 0 .
$$

2. If $v^{*}\left(x_{0}, t_{0}\right)>0$ and since $v^{*}$ is a subsolution of (5.2), $\left[\partial_{t} \phi-\mathcal{A}^{0} \phi\right]\left(x_{0}, t_{0}\right) \leq 0$. Thus we may assume that $v^{*}\left(x_{0}, t_{0}\right)=0$ and $|D \phi|\left(x_{0}, t_{0}\right) \neq 0$.

Lemma 5.5 yields $v^{0} \leq v_{*} \leq v^{*}$ and so $v^{0} \leq \phi_{+}$in $\Sigma$ and $v^{0}\left(x_{0}, t_{0}\right)=\phi_{+}\left(x_{0}, t_{0}\right)=$ 0 . Moreover, Lemma 5.6 (ii) implies that $\left(x_{0}, t_{0}\right) \in \Gamma\left(v^{0}\right)$. Since $v^{0}$ is a subsolution of $\left(P_{0}\right)$, it follows that

$$
\min \left(\left(\partial_{t} \phi-\mathcal{A}^{0} \phi\right)\left(x_{0}, t_{0}\right),\left(\phi_{t}-F^{0}(D \phi)|D \phi|\right)\left(x_{0}, t_{0}\right)\right) \leq 0,
$$

which proves the claim, and thus completes the proof.

Proof of Theorem 5.2 .

1. Arguing as in the proof of Lemma 3.8, we obtain

$$
v^{*}(x, 0)=v_{0}(x) .
$$

Since $v^{*}$ is a subsolution and $v^{0}$ is a supersolution of $(P)$, Corollary 3.12 yields

$$
v^{*}(x, t) \leq v^{0}(x, t) .
$$

Since $v^{0} \leq v_{*}$, we obtain $v_{*}=v^{*}=v^{0}$. In particular $\Gamma\left(v^{0}\right)=\Gamma\left(v^{*}\right)$ and $v^{\varepsilon}$ locally uniformly converges to $v^{0}$. 
2. It remains to show the uniform convergence of the free boundaries. Suppose $\left(x_{0}, t_{0}\right) \in \Gamma\left(v^{0}\right)$. Since we know now that $v^{\varepsilon}$ locally uniformly converges to $v^{0}$, arguing as in the proof of Lemma 5.6 (i) with the whole sequence $\varepsilon$ yields that

$$
d\left(\left(x_{0}, t_{0}\right), \Gamma\left(v^{\varepsilon}\right)\right) \leq \delta \text { if } \varepsilon<\varepsilon_{0} .
$$

On the other hand if $\left(x_{\varepsilon}, t_{\varepsilon}\right) \in \Gamma\left(v^{\varepsilon}\right)$ with $t_{\varepsilon} \leq T$, then Lemma 5.4 and the compactness of the sets $\Gamma\left(v^{0}\right) \cap\{0 \leq t \leq T\}$ and $\Gamma\left(v^{\varepsilon}\right) \cap\{0 \leq t \leq T\}$ yield that for any $\delta>0$ there exists $\varepsilon_{0}>0$ such that

$$
d\left(\Gamma\left(v^{0}\right),\left(x_{\varepsilon}, t_{\varepsilon}\right)\right) \leq \delta \text { if } \varepsilon<\varepsilon_{0} .
$$

The two inequalities above yield the local uniform convergence of $\Gamma\left(v^{\varepsilon}\right)$ to $\Gamma\left(v^{0}\right)$ with respect to the Hausdorff distance.

\section{Appendix A. Construction of Barriers}

In this appendix, we detail the construction of barriers that are used in various proofs in the paper. The aim is to construct a solution of $\mathcal{A} h=n$ supported in a unit ball (when $\mathcal{A}=\Delta$, this is given by $x^{2} / 2$ ). For that, we need to find $h$ such that

$$
\Sigma_{j} a_{i j}(x) \partial_{x_{j}} h=x_{i} \quad \text { for } 1 \leq i \leq n .
$$

This equality can also be written (in matrix form) as $A \cdot D h=x$ (with $A=\left(a_{i j}\right)$ ). Since $A$ is positive definite,

$$
D h=A^{-1} x .
$$

It is now readily seen that this equation has a unique solution for any given value of $h(0)$. Furthermore, note that $h(x)$ has a quadratic growth rate:

$$
h(x)-h(0)=\int_{0}^{|x|} \nu \cdot A^{-1}(r \nu) d r \in\left[(\Lambda)^{-2} \lambda|x|^{2},(\lambda)^{-2} \Lambda|x|^{2}\right],
$$

where $\nu=\frac{x}{|x|}$. The second inequality follows since

$$
\lambda(\Lambda)^{-2} \leq \lambda\left|A^{-1} \nu\right|^{2} \leq \nu \cdot A^{-1} \nu \leq \Lambda\left|A^{-1} \nu\right|^{2} \leq(\lambda)^{-2} \Lambda
$$

for any unit vector $\nu \in \mathbb{R}^{n}$.

Note also that the level sets of $h$ are strictly convex since $D^{2} h=A^{-1}$ is positive definite.

Suppose now that $B^{(n+1)}$ is a space-time ball which touches $(0,0)$ on its boundary, away from the top and bottom portions of the ball.

By considering functions of the form

$$
\varphi(x, t)=\left(a n\left(t-t_{0}\right)-a h\left(x-x_{0}\right)+b\right)_{+},
$$

where $a, b$ are constants, and by dilation, one can generate local barriers which solve

$$
\begin{cases}\varphi_{t}-\mathcal{A} v=0 & \text { in } S:=\{\varphi>0\}, \\ |D \varphi|\left(x_{0}, t_{0}\right)=c & \text { at }(0,0), \\ \partial S \text { is } C^{2,1} \text { with }(0,0) \in \partial S, & \\ S \subset B^{(n+1)} \text { or } S \subset\left(B^{(n+1)}\right)^{c} . & \end{cases}
$$


Using this family of barriers, one can now proceed as in the proof of Theorem 2.2 in [K1] to establish the comparison principle (Theorem 2.8) for the viscosity solutions of $(P)$.

Proof of Lemma 3.6.

1. The proof relies on the construction of the barrier and the comparison principle for the obstacle problem.

For this purpose we define

$$
\varphi(x, t)=(f(t)-h(x)+b)_{+},
$$

where $b$ is large enough such that $v_{0}(x) \leq(-h(x)+b)_{+}$and $f(t)$ is an increasing function of $t$ satisfying

$$
f(0)=0, \quad f^{\prime}(t) \geq \Lambda \sup \left\{|D h|^{2}(x): h(x)=f(t)+b\right\} .
$$

Clearly, if such a function $f(t)$ can be constructed, then $\varphi(x, t)$ is a classical supersolution of $(P)$, and so the solution $u$ of (2.1) will be below $\int_{0}^{t} \varphi(x, s) d s$. Lemma 3.6 then follows with

$$
M(T)=\sup \{x: h(x) \leq b+f(T)\}<\infty .
$$

2. It remains to check that $f(t)$ exists. Due to (A.1), we have

$$
c|x|^{2} \leq h(x) \leq C|x|^{2} .
$$

Hence if $h(x)=f(t)+b$, then $|x|^{2} \sim f(t)+b$ and thus

$$
|D h(x)|^{2}=\left|A^{-1} x\right|^{2} \leq C(f(t)+b),
$$

where $C$ depends on $\lambda$ and $\Lambda$. Therefore $f$ must solve

$$
f^{\prime}(t)=C(f(t)+b), f(0)=0,
$$

which leads to $f(t)=b\left(e^{C t}-1\right)$.

\section{Appendix B. Further Results on the Parabolic obstacle problem}

Let $\mathcal{O}$ be a bounded open subset of $\mathbb{R}^{n}$ and let $\mathcal{K}=\left\{v \in H_{0}^{1}(\mathcal{O}) ; v \geq 0\right\}$. We consider the variational inequality

$$
\begin{aligned}
& u \in L^{2}\left(0, T, H^{2}(\mathcal{O})\right), \quad u_{t} \in L^{2}\left(0, T ; L^{2}(\mathcal{O})\right), \\
& \left(u_{t}-\mathcal{A} u\right)(v-u) \geq f(v-u) \quad \text { for all } v \in \mathcal{K}, \\
& u(t) \in \mathcal{K}, \quad u(x, 0)=0,
\end{aligned}
$$

where $\mathcal{A}$ is a uniformly elliptic operator and

$$
f(x)= \begin{cases}v_{0}(x) & \text { in } \Omega_{0}, \\ -1 & \text { in } \mathbb{R}^{n} \backslash \Omega_{0} .\end{cases}
$$

We show here the comparison principle and stability results that are used in this paper. 
Lemma B.1 (Comparison principle). Let $f_{1} \leq f_{2}$ and denote by $u_{1}$ and $u_{2}$ the corresponding solutions of (B.1). Then $u_{1} \leq u_{2}$ in $\mathcal{O} \times(0, \infty)$.

Proof. The proof follows from [FK]. We recall it here for the sake of completeness: Integrating (B.1) yields, for any $v \in H^{1}(\mathcal{O} \times(0, T))$ satisfying $v \geq 0$ and $v(\cdot, 0)=0$,

$$
\iint a_{i j} D_{j} u_{2} D_{i}\left(v-u_{2}\right)+\partial_{t} u_{2}\left(v-u_{2}\right) d x d t \geq \iint f_{2}\left(v-u_{2}\right) d x d t
$$

and if we take $v=\max \left(u_{1}, u_{2}\right)$, we get

$$
\iint_{B} a_{i j} D_{j} u_{2} D_{i}\left(u_{1}-u_{2}\right)+\partial_{t} u_{2}\left(u_{1}-u_{2}\right) d x d t \geq \iint_{B} f_{2}\left(u_{1}-u_{2}\right) d x d t,
$$

where $B=\left\{u_{1}>u_{2}\right\}$. Denoting $\xi=\left(u_{1}-u_{2}\right) \chi_{\left\{u_{1}>u_{2}\right\}} \in H^{1}(D)$, we can also write:

$$
\iint a_{i j} D_{j} u_{2} D_{i} \xi+\partial_{t} u_{2} \xi d x d t \geq \iint f_{2} \xi d x d t
$$

Next, we note that $u_{1}>u_{2} \geq 0$ in $B$ and so $\partial_{t} u_{1}-\mathcal{A} u_{1}=f_{1}$ in $B$. We deduce

$$
-\iint a_{i j} D_{j} u_{1} D_{i} \xi+\partial_{t} u_{1} \xi d x d t=-\iint f_{1} \xi d x d t .
$$

Adding those last two equations and using the definition of $\xi$, we deduce

$$
-\iint a_{i j} D_{j} \xi D_{i} \xi d x d t-\int \frac{1}{2} \xi^{2}(x, T) d x \geq-\iint\left(f_{1}-f_{2}\right) \xi d x d t .
$$

Since $\xi \geq 0$ and $f_{1}-f_{2} \leq 0$, it follows that $\xi=0$ a.e., i.e. $|B|=0$.

Lemma B.2 (Stability). Let $f_{1}$ and $f_{2}$ be two functions and denote by $u_{1}$ and $u_{2}$ the corresponding solutions of (B.1). Then

$$
\left\|u_{1}-u_{2}\right\|_{L^{\infty}} \leq C|| f_{1}-f_{2} \|_{L^{n+1}} .
$$

Proof. Let $w_{1}$ (respectively $w_{2}$ ) be the solution of (B.1) associated with $g_{1}=$ $\min \left(f_{1}, f_{2}\right)$ (respectively $g_{2}=\max \left(f_{1}, f_{2}\right)$ ). The previous lemma implies that

$$
w_{1} \leq u_{i} \leq w_{2} \text { for } i=1,2,
$$

so it suffices to prove that the lemma holds for $w_{1}$ and $w_{2}$. Let $\Phi$ be a solution of

$$
\Phi_{t}-\mathcal{A} \Phi=g_{2}-g_{1}
$$

in $\mathcal{O} \times(0, T)$ with $\Phi(x, 0)=0$ and $\Phi(x, t)=0$ on $\partial \mathcal{O}$. The function $w_{3}=w_{1}+\Phi$ satisfies

$$
\partial_{t} w_{3}-A w_{3} \geq g_{2} .
$$

This inequality is enough to carry through the argument of the previous lemma with $u_{1}=w_{2}$ and $u_{2}=w_{3}$. It follows that

$$
w_{1} \leq w_{2} \leq w_{1}+\Phi .
$$


Finally, the classical estimate for a parabolic equation with bounded measurable coefficients (see, for instance Aronson [A]) yields

$$
\sup _{\mathcal{O} \times(0, T)} \Phi \leq C(\mathcal{O})\left\|g_{2}-g_{1}\right\|_{L^{n}}
$$

which completes the proof.

\section{REFERENCES}

[AK] M. A. Akcoglu and U. Krengel, Ergodic theorems for superadditive processes, J. Reine Angew. Math., 323 (1981), 53-67. MR611442 (83k:28015)

[A] D. G. Aronson, On the Green's function for second order parabolic differential equations with discontinuous coefficients. Bull. Amer. Math. Soc. 69 (1963), 841-847. MR0155109 $(27: 5049)$

[BLP] A. Bensoussan, J.-L. Lions and G. Papanicolaou. Asymptotic analysis for periodic structures. Studies in Mathematics and its Applications, North-Holland, Amsterdam-New York, 1978. MR503330 (82h:35001)

[CF] L. Caffarelli and A. Friedman, Continuity of the temperature in the Stefan problem, Indiana U. Math. J. 28 (1979) no.1, 53-70. MR.523623 (80i:35104)

[CL] G. M. Crandall and P.-L. Lions, Viscosity solutions of Hamilton-Jacobi equations. Trans. Amer. Math. Soc. 277 (1983), 1-42. MR690039 (85g:35029)

[Da] G. Dal Maso, An introduction to $\Gamma$-convergence. Progress in Nonlinear Differential Equations and their Applications, Birkhäuser Boston (1993). MR.1201152 (94a:49001)

[Do] J. Doob, Classical potential theory and its probabilistic counterpart, New-York, SpringerVerlag, 1984. MR 731258 (85k:31001)

[DM1] G. Dal Maso and L. Modica, Nonlinear stochastic homogenization, Ann. Mat. Pura Appl. 144 (1986), 347-389. MR870884 (88h:49025)

[DM2] G. Dal Maso and L. Modica, Nonlinear stochastic homogenization and ergodic theory, J. Reine Angew. Math., 368 (1986), 28-42. MR850613 (88k:28021)

[Du] G. Duvaut, Résolution d'un problème de Stefan (fusion d'un bloc de glaceà zéro degreé). C. R. Acad. Sci. Paris Ser. A-B 276 (1973), A1461-A1463. MR0328346 (48:6688)

[F] A. Friedman, The Stefan problem in several space variables. Trans. Amer. Math. Soc. 133 (1968) 51-87. MR0227625 (37:3209)

[FK] A. Friedman and D. Kinderlehrer. A one phase Stefan problem. Indiana Univ. Math. J. 24 (1974/75), 1005-1035. MR0385326 (52:6190)

[GL] N. Garofalo and E. Lanconelli, Wiener's criterion for parabolic equations with variable coefficients and its consequences. Trans. Amer. Math. Soc. 308 (1988), no. 2, 811-836. MR.951629 (89k:35104)

[H] E.-I. Hanzawa, Classical solutions of the Stefan problem. Tôhoku Math. J. 33 (1981), 297-335. MR633045 (82k:35065)

[Ka] S. L. Kamenomostskaja, On Stefan's problem. (Russian) Mat. Sb. (N.S.) 53 (1961) 489-514. MR0141895 (25:5292)

[K1] I. Kim, Uniqueness and Existence result of Hele-Shaw and Stefan problem, Arch. Rat. Mech. Anal, 168 (2003), 299-328. MR.1994745 (2004k:35422)

[K2] I. Kim, Homogenization of the free boundary velocity, Arch. Rat. Mech. Anal, 185 (2007), 69-103. MR2308859 (2008f:35019)

$[\mathrm{KM}]$ I. Kim and A. Mellet, Homogenization of a Hele-Shaw problem in periodic and random media, submitted.

[M] J. Moser, On Harnack's inequality for parabolic differential equations, Comm. Pure Appl. Math. 17 (1964), 101-134. MR0159139 (28:2357)

[O] O. A. Oleĭnik, A method of solution of the general Stefan problem. Dokl. Akad. Nauk SSSR 135 1054-1057 (Russian); translated as Soviet Math. Dokl. 1 (1960) 1350-1354. MR 0125341 (23:A2644)

[P] M. Primicerio, Stefan-like problems with space-dependent latent heat, Meccanica 5 (1970), 187-190. MR0372424 (51:8633)

[PV] G. Papanicolaou and S.R.S. Varadhan, Diffusions with random coefficients. Statistics and probability: Essays in honor of C. R. Rao, pp. 547-552, North-Holland, Amsterdam, 1982. MR659505(85e:60082) 
[R1] J.-F. Rodrigues, The Stefan problem revisited. Mathematical models for phase change problems (ibidos, 1988), 129-190, Internat. Ser. Numer. Math., 88, Birkhäuser, Basel, 1989. MR.1038069 (91e:80008)

[R2] J.-F. Rodrigues, Free boundary convergence in the homogenization of the one-phase Stefan problem, Trans. Amer. Math. Soc. 274 (1982), no.1, 297-305. MR670933 (83k:35084)

[Rou] T. Roubíček, The Stefan problem in heterogeneous media, Ann. Inst. H. Poincaré Anal. Non Linéaire, 6 (1989), 481-501. MR1035339 (91c:35167)

Department of Mathematics, University of California, los Angeles, Los Angeles, CALIFORNIA 90095

Department of Mathematics, University of Maryland, College Park, Maryland 20742 